JGR Oceans

\author{
RESEARCH ARTICLE \\ 10.1029/2019JC015229 \\ Key Points: \\ - Submesoscale frontal eddies are \\ generated due to barotropic \\ instability in the Agulhas Current \\ - The background strain provides \\ favorable conditions for instability \\ generation \\ - A new regime of variability is \\ highlighted in addition to the two \\ modes described by Paldor and \\ Lutjeharms (2009)
}

Correspondence to:

P. Tedesco,

ptedesco@ifremer.fr

Citation:

Tedesco, P., Gula, J., Ménesguen, C., Penven, P., \& Krug, M. J. (2019). Generation of submesoscale frontal eddies in the Agulhas Current. Journal Geophysical Research: Oceans, 124, 7606-7625. https://doi.org/10. 1029/2019JC015229

Received 16 APR 2019

Accepted 9 SEP 2019

Accepted article online 12 SEP 2019

Published online 12 NOV 2019

\section{Generation of Submesoscale Frontal Eddies in the Agulhas Current}

\author{
P. Tedesco ${ }^{1}\left(\mathbb{D}\right.$, J. Gula $^{1}(\mathbb{D})$, C. Ménesguen ${ }^{1}$, P. Penven $^{1}\left(\mathbb{D}\right.$, and M. Krug and $^{2,3,4}$ \\ ${ }^{1}$ Université de Bretagne Occidentale, CNRS, IRD, Ifremer, Laboratoire d'Océanographie Physique et Spatiale (LOPS), \\ IUEM, Brest, France, ${ }^{2}$ Council for Scientific and Industrial Research, Natural Resources and the Environment, Cape \\ Town, South Africa, ${ }^{3}$ Department of Oceanography, Ma-re Institute, University of Cape Town, South Africa, \\ ${ }^{4}$ Nansen-Tutu Centre for Marine Environmental Research, Department of Oceanography, University of Cape Town, \\ South Africa
}

\begin{abstract}
This study addresses the dynamics of the Agulhas inshore front in the submesoscale range upstream of $26^{\circ}$ E. Submesoscale frontal eddies are observed in the vicinity of Port Elizabeth $\left(26^{\circ} \mathrm{E}\right)$ from satellite images and in observations collected from underwater gliders. Using a submesoscale-resolving numerical model $(\mathrm{dx} \sim 0.75 \mathrm{~km})$, we are able to simulate similar submesoscale eddies. Barotropic instability is confirmed as the generation mechanism by a one-dimensional linear stability analysis and an eddy kinetic energy budget. Kinetic energy is transferred from the mean flow to the eddies through the mean horizontal shear, which is a signature of barotropic instability. When the Agulhas Current is in a nonmeandering state, submesoscale eddy generation is a recurrent process which locally drives the front's variability. Along the front, the spatial variability of barotropic instability is shaped by the background strain. A large strain aligned with the frontal axis intensifies the frontal shear upstream of $28^{\circ} \mathrm{E}$ while a weakening of the strain allows for barotropic instability to be triggered downstream. Although an intermittent process, the barotropic instability shows a dominant period of variability comparable with the variability of the Agulhas Current and Undercurrent.
\end{abstract}

\section{Introduction}

The Agulhas Current is the most intense western boundary current of the South Hemisphere with a mean transport of $\sim 80 \mathrm{~Sv}$ (Beal et al., 2015). It flows poleward along the South African coastline between $32^{\circ} \mathrm{E}$ and $19^{\circ} \mathrm{E}$ (Lutjeharms, 2006) and forms part of the South Indian Ocean subtropical gyre (Figure 1). The Agulhas Current finds its origin in the Mozambique Channel and from southeast Madagascar. Between about $19^{\circ} \mathrm{E}-20^{\circ} \mathrm{E}$, the Agulhas Current retroflects and flows eastward back into the south Indian Ocean as the Agulhas Return Current. Large anticyclones, named Agulhas Rings, which are shed at the Agulhas Retroflection, transport warm and salty waters into the South Atlantic Ocean (Lutjeharms \& Gordon, 1987). This heat and salt flux, named the Agulhas leakage, is thought to be a key component of the upper branch of the global overturning circulation (Beal et al., 2011; Peeters et al., 2004; Weijer et al., 2002).

The Agulhas Current is described as spatially "bi-modal" (Lutjeharms, 2006; Paldor \& Lutjeharms, 2009) with a stable branch upstream of Port Elizabeth $\left(26^{\circ} \mathrm{E}\right)$ and an unstable branch downstream.

Upstream of Port Elizabeth $\left(26^{\circ} \mathrm{E}\right)$, the jet is narrow and flows close to a steep and straight topography. The Agulhas Current in this region is occasionally disturbed by the passage of Natal Pulses (Figure 1), solitary meanders with diameters of 50-200 km developing near the Natal Bight ( 31 ${ }^{\circ}$ E, Figure 1) four to five times a year and traveling downstream (Lutjeharms, 2006; Schouten et al., 2002). Due to dissipation and/or merging processes, only a fraction of Natal Pulses (around 1.7 per year) reaches the eastern margin of the Agulhas Bank ( $24^{\circ}$ E, Figure 1) (Krug \& Tournadre, 2012; Krug et al., 2014; Rouault \& Penven, 2011).

Downstream of Port Elizabeth $\left(26^{\circ} \mathrm{E}\right)$, the current becomes increasingly unstable. In addition to Natal Pulses, frontal eddies (Schumann \& Van Heerden, 1988), also called shear-edge eddies (Lutjeharms et al., 1989), with a diameter of 50-100 km, develop along the eastern margin of the Agulhas Bank (Figure 1). Further downstream, cyclonic eddies are generated from the detachment of the Agulhas Current at the tip of the Agulhas Bank (Penven et al., 2001). All these meanders and eddies are associated with features such as filaments and plumes which can intrude onto the continental shelf (Krug et al., 2014; Lutjeharms, 2006).
(C)2019. American Geophysical Union. All Rights Reserved. 


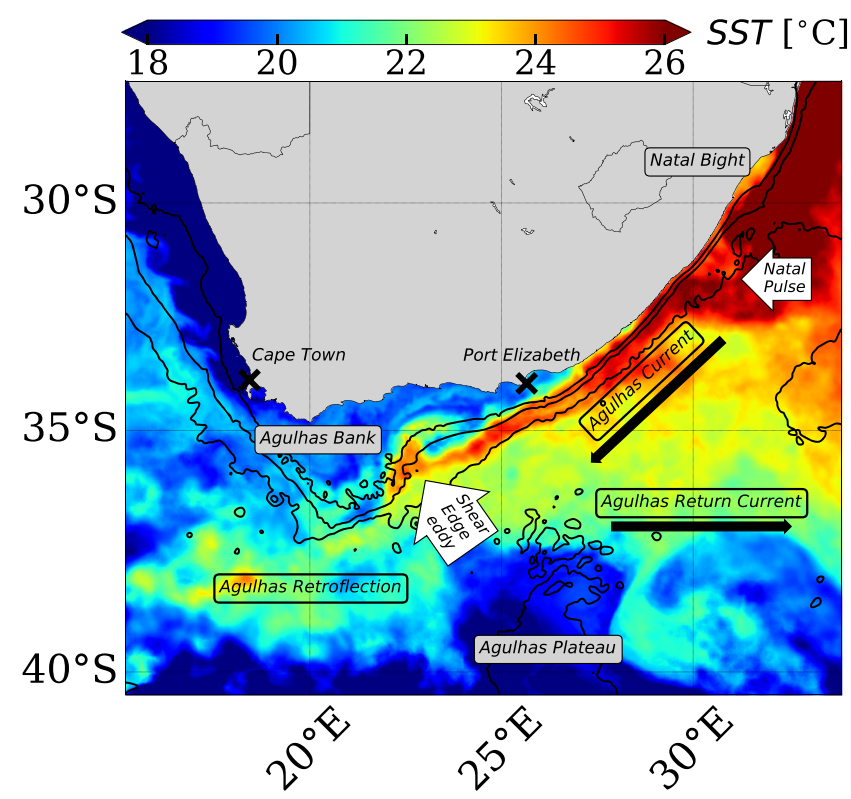

Figure 1. Snapshot of the Sea Surface Temperature (SST) $\left[{ }^{\circ} \mathrm{C}\right]$ on 4 June 2014 from the Odyssea Regional South African product, showing the warm Agulhas Current extending from the southern boundary of the Mozambique Channel to the retroflection and returning in the South Indian gyre as the Agulhas Return Current (black legends). The main topographic characteristics along the Agulhas path are displayed (gray legends). The thin black lines follow the 200, 1000, and $3000 \mathrm{~m}$ isobaths. Two perturbations, a Natal Pulse and a Shear-Edge eddy (white arrows), are developing along the Agulhas cyclonic front.

The Agulhas retroflection $\left(20^{\circ} \mathrm{E}-19^{\circ} \mathrm{E}\right.$, Figure 1) has a high level of eddy variability due to the spawning of Agulhas Rings (Lutjeharms \& Gordon, 1987).

The first baroclinic Rossby radius of deformation $(R d)$ is $O(20-40) \mathrm{km}$ in the region (Chelton et al., 1998). As the features described above are typically larger than $R d$, Lutjeharms (2006) and Paldor and Lutjeharms (2009)'s historical descriptions of the Agulhas Current dynamics accounts for the mesoscale range. However, the Shelf Agulhas Glider Experiment (SAGE) (Krug et al., 2017) provides recent observations of submesoscale instabilities $(\mathrm{L}<R d)$ in the region. In April 2015, two Seagliders sampled submesoscale frontal cyclonic eddies, with diameters $\sim 15 \mathrm{~km}$, offshore Port Elizabeth (Figure 2a). Features similar to the SAGE's eddies are regularly visible on maps of Sea Surface Temperature (SST). Figure 2b shows submesoscale meanders along a portion of the cyclonic inshore front $\left(28.5^{\circ} \mathrm{E}-26^{\circ} \mathrm{E}\right)$, just upstream of Port Elizabeth.

Observations of these frontal submesoscale eddies give a new insight on the historical description of the Agulhas Current dynamics (Lutjeharms, 2006; Paldor \& Lutjeharms, 2009). They show features at scales smaller that the previously documented at a location where the current was reported "stable." The investigation of these submesoscale features should give a more comprehensive understanding of the Agulhas Current frontal dynamics. As a consequence of, developing at the Agulhas inshore front, these submesoscale eddies can influence the shelf dynamics and variability. Plumes, filaments, and eddy shedding affect the shelf by driving horizontal water masses transport (Krug et al., 2014; Lutjeharms, 2006) and so might do the frontal submesoscale eddies. In addition, the ageostrophic components of the submesoscale frontal flow lead to large vertical velocities and enhanced vertical exchanges, which can impact biogeochemistry and the primary production of the upper ocean (Mahadevan, 2014).

Similar examples of submesoscale frontal cyclonic eddies are reported for two other western boundary currents. In the Gulf Stream, eddies with diameters of 10-15 km were observed on satellite SST images and modeled using a submesoscale-resolving simulation (Gula et al., 2015b). In the Eastern Australian Current, eddies with diameters of $10 \mathrm{~km}$ were observed with coastal HF radars and satellites (Schaeffer et al., 2017). In addition, frontal submesoscale eddies may also be of interest for the ocean energy budget as they can trigger an energy route from the large-scale geostrophic circulation toward smaller scales (Ferrari \& Wunsch, 2010). 

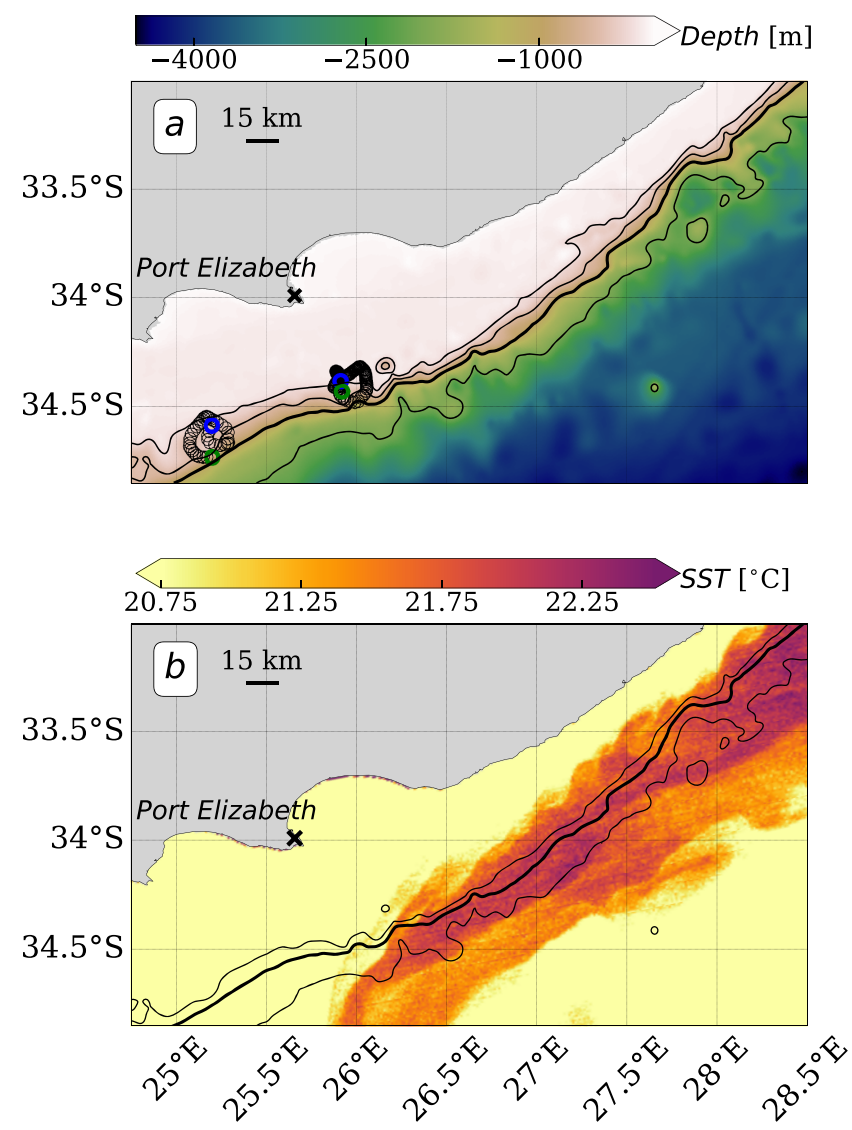

Figure 2. (a) Black circles track two Seagliders when they sampled two submesoscale cyclonic features between the 19 April 2015 and the 20 May 2015 (Krug et al., 2017). The first and last measures inside these features are respectively marked with green and blue circles. The background colors represent the bathymetry $[\mathrm{m}]$. The thin black lines follow the 200, 500, and $2000 \mathrm{~m}$ isobaths. The bold black line is the $1000 \mathrm{~m}$ isobath, which corresponds to the Agulhas front location during the SAGE observations. (b) Snapshot of Sea Surface Temperature (SST) $\left[{ }^{\circ} \mathrm{C}\right]$, from the denoised Moderate-Resolution Imaging Spectroradiometer (MODIS) on 19 July 2010. The black lines represent the same isobaths than in (a), except for the $200 \mathrm{~m}$ which is not represented. Submesoscale meanders develop upstream of Port Elizabeth between $28.5^{\circ} \mathrm{E}$ and $26^{\circ} \mathrm{E}$.

In this study, we address the dynamics of the Agulhas inshore front around Port Elizabeth ( $\left.26^{\circ} \mathrm{E}\right)$ using a submesoscale-resolving numerical model. We aim to characterize the frontal submesoscale eddy generation mechanism, its spatial and time variability, and the factors involved in their triggering. In section 2, we present the numerical simulations. In section 3, we compare the modeled mean dynamics of the Agulhas Current with in situ and satellite observations. In section 4, we investigate the generation mechanism of the submesoscale eddies in the model. Finally, in section 5 we discuss the spatial and time variability of the submesoscale eddy generation mechanism.

\section{Simulation Setup}

The realistic simulations are run with the CROCO (Coastal and Regional Ocean COmmunity model) model (Debreu et al., 2012), which is based on ROMS (Shchepetkin \& McWilliams, 2005). This model solves the hydrostatic primitive equations for the momentum and the state variables and uses topographic following vertical coordinates.

To set up a submesoscale eddy-resolving simulation, we use a nesting approach. Four nests with respective horizontal resolutions of $\mathrm{dx} \sim 22.5,7.52 .5$, and $0.75 \mathrm{~km}$ are embedded (Figure 3). The highest-resolution grid ( $\mathrm{dx} \sim 0.75 \mathrm{~km}$ ) has a sufficient effective horizontal resolution (Soufflet et al., 2016) to solve the submesoscale range $(<O(30) \mathrm{km})$ in the Agulhas region. 


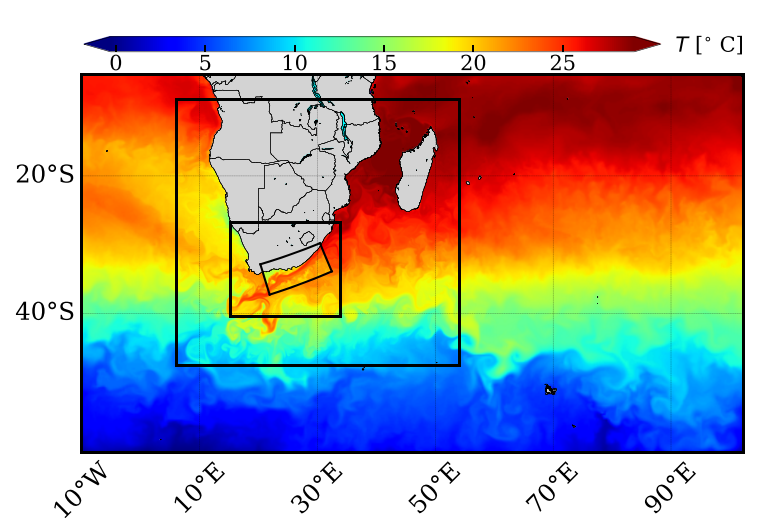

Figure 3. Snapshot of surface temperature $\left[{ }^{\circ} \mathrm{C}\right]$ simulated by CROCO for the four nested domains : $\mathrm{dx} \sim 22,7.5,2.5$, and $0.75 \mathrm{~km}$.
The three first nests $(\mathrm{dx} \sim 22,7.5$, and $2.5 \mathrm{~km})$ are set up with the same nesting procedure, forcings, and vertical grid. The nesting procedure is a two-way online nesting based on AGRIF (Debreu et al., 2012). At each time step each "child" grid is forced by its "parent" grid, and feedbacks from the "child" grids (finer scales) to larger grids (larger scales) are taken into account. The GLORYS oceanic reanalysis (Ferry et al., 2012) provides the lateral boundary conditions for the largest grid $(\mathrm{dx} \sim 22 \mathrm{~km})$. The surface forcings are provided by a bulk formulation (Fairall et al., 1996) using the daily ERA-ECMWF's reanalysis (Dee et al., 2011). Relative winds are preferred to absolute winds due to their implications in a more realistic dissipation of mesoscale eddies in the Agulhas Current (Renault et al., 2017). The bathymetry is built from the General Bathymetric Chart of the Oceans 30-s resolution (Weatherall et al., 2015). The topography is locally smoothed when steepness exceeds the threshold $r_{\max }=0.2$, with $r_{\max }=\frac{\nabla h}{h}$ the slope parameter defined by Beckmann and Haidvogel (1993). The vertical grids are designed following the Haidvogel and Beckmann (1999) method. Parameters driving the levels refinement at the surface and the bottom are respectively $\theta_{s}=5, \theta_{b}=0$. The transition depth between flat-z levels and terrain following sigma level is set to $h_{\text {cline }}=10 \mathrm{~m}$.

The highest-resolution grid $(\mathrm{dx} \sim 0.75 \mathrm{~km})$ set up differs from the three first grids on several points. The nesting procedure is an off-line one-way nesting as described by Gula et al. (2015a). In this case, the parent forces the child grid without feedback from the finer toward the larger scales. The surface forcing and the bathymetry are derived from the same data sets as for parent grids. The boundary conditions are provided by the nearest parent grid $(\mathrm{dx} \sim 2.5 \mathrm{~km})$ at a daily rate. The vertical resolution is increased relative to the horizontal resolution with 100 vertical levels. They are distributed following Lemarié et al. (2012) method with parameters $\theta_{s}=6, \theta_{b}=4$ and $h_{\text {cline }}=300 \mathrm{~m}$.

The horizontal advection of momentum is discretized using a third-order upstream biased numerical scheme and horizontal advection of tracers is discretized using a split and rotated third-order upstream-biased numerical scheme (Marchesiello et al., 2009). The later scheme reduces spurious diapycnal mixing. On the vertical, the momentum advection scheme is semi-implicit, with an adjustment between explicit and implicit formulation according to the model stability.

A K-profile parameterization (Large et al., 1994) parameterizes the vertical mixing of tracers and momentum. The adjusted K-profile parameterization by Durski et al. (2004) is used for the bottom boundary layer. Both parameterizations take into account the Lemarie et al.'s (2012) modification of the Richardson number criterion with the critical value $\left(R i_{c r}=0.15\right)$. The bottom viscous stress has a quadratic form.

The parent grid $(\mathrm{dx} \sim 22.5 \mathrm{~km}$ has $450 \times 258 \times 60$ points $)$ covers most of the South Equatorial Indian Ocean and a part of the Atlantic Ocean. The first child grid $(\mathrm{dx} \sim 7.5 \mathrm{~km})$, with $578 \times 542 \times 60$ points, encompasses the African continent from the Equator to the Antarctic Circumpolar Current. It includes the Mozambique Channel, where large anticyclones are generated (Halo et al., 2014) and related to Natal Pulses development in the Agulhas Current (Elipot \& Beal, 2015). Both nests are spun-up from their initial state for 3 years. The third child grid $(\mathrm{dx} \sim 2.5 \mathrm{~km})$, with $674 \times 578 \times 60$ points, encompasses the Agulhas Current from upstream the Natal Bight $\left(\sim 31^{\circ} \mathrm{E}\right)$ to the Agulhas retroflection region $\left(\sim 19^{\circ} \mathrm{E}-20^{\circ} \mathrm{E}\right)$. This simulation is spun up for a year and run from 1993 to 2014 . The highest-resolution grid ( $\mathrm{dx} \sim 0.75 \mathrm{~km}$ has $1352 \times 652 \times 100$ points) is centered on the SAGE area (Port Elizabeth, $26^{\circ} \mathrm{E}$ ) and resolves more precisely the submesoscale dynamics of the current and their potential interactions with the topography. This grid is run from 01/09/1995 to 31/03/1997. This period is centered on the year 1996, during which no upstream Agulhas retroflection is observed or simulated.

\section{Observed and Modeled Agulhas Dynamics}

In this section, we evaluate the mean Agulhas Current dynamics. First, we compare the mean modeled surface Agulhas Current to satellite data, and second, we compare the vertical and the surface structures of the mean modeled Agulhas inshore front, in the vicinity of our area of interest (Port Elizabeth, $26^{\circ} \mathrm{E}$ ) with in situ data. 


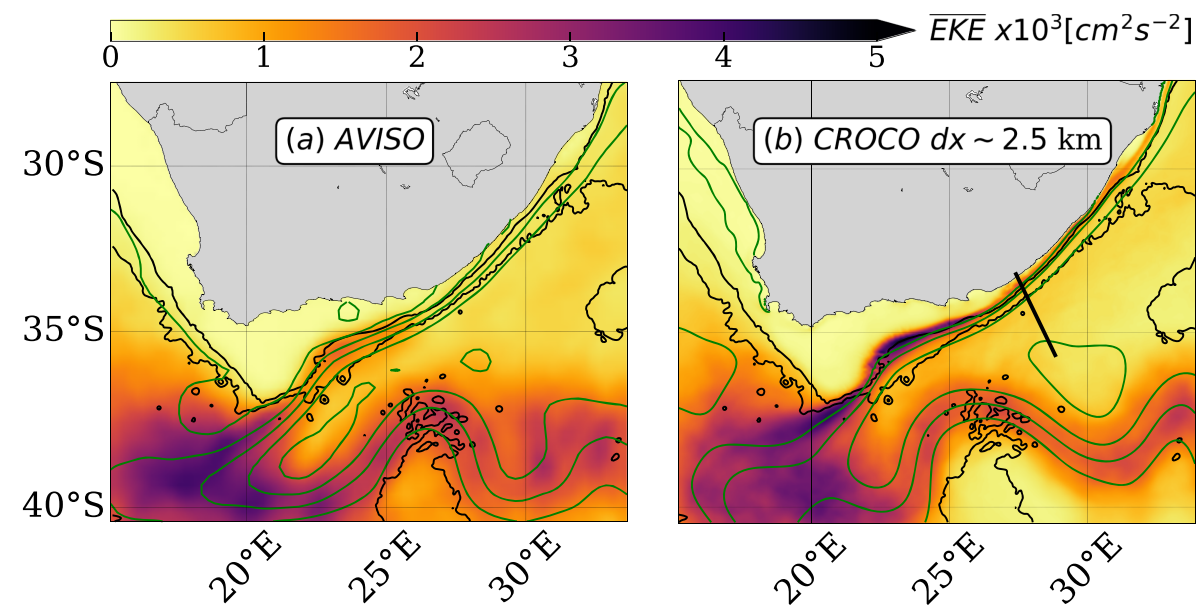

Figure 4. Maps of the surface $\overline{E K E}\left[\mathrm{~cm}^{2} \mathrm{~s}^{-2}\right.$ ] for (a) AVISO and for (b) CROCO at dx $2.5 \mathrm{~km}$ both for the 1993-2003 period. Black contours are the 1000 and $3000 \mathrm{~m}$ isobaths, and the green contours are the isolines of mean sea surface height between -2 and $2 \mathrm{~m}$ with a lag of $0.25 \mathrm{~m}$. The black segment in Figure $4 \mathrm{~b}$ shows the location of the vertical sections plotted along the ACT array in Figures 5. Both observed and modeled surface $E K E$ have low $\left(O(0.5-2) \times 10^{-3}\right.$ $\left.\mathrm{cm}^{2} \mathrm{~s}^{-2}\right)$ and large $\left(>O(3.5) \times 10^{-3} \mathrm{~cm}^{2} \mathrm{~s}^{-2}\right)$ values along the upstream stable and downstream unstable Agulhas branches. The topography plays a role on the current dynamics and small scales $(10-100 \mathrm{~km})$ can locally drive the front variability.

\subsection{The Mean Agulhas Current: Surface Description}

The mean Eddy Kinetic Energy $(\overline{E K E})$ is defined as $\overline{E K E}=0.5\left(\overline{u^{\prime 2}}+\overline{v^{\prime 2}}\right)$ with $u$ and $v$ the horizontal velocity components. The overbar denotes a time mean and the prime denotes fluctuations relative to this mean. We compare the surface $\overline{E K E}$ from the $\mathrm{dx} \sim 2.5 \mathrm{~km}$ nest with the one derived from satellite altimetry observations of absolute dynamic topography gridded at $1 / 4^{\circ}$ provided by AVISO (Figure 4). Both observed and modeled $\overline{E K E}$ are defined from geostrophic velocity anomalies diagnosed from a 10-year long subset (1993-2003) of sea surface height anomaly.

The observed and modeled surface $\overline{E K E}$ patterns match the spatially "bi-modal" dynamics of the Agulhas Current (Figures $4 \mathrm{a}$ and $4 \mathrm{~b}$ respectively). Upstream of Port Elizabeth $\left(26^{\circ} \mathrm{E}\right), \overline{E K E}$ is low $\left(O(0.5-2) \times 10^{3}\right.$ $\left.\mathrm{cm}^{2} \mathrm{~s}^{-2}\right)$, and downstream it is more intense $\left(O>(3.5) \times 10^{3} \mathrm{~cm}^{2} \mathrm{~s}^{-2}\right)$. The Agulhas retroflection region, where Agulhas Rings and Agulhas Eddies are generated, has the largest values of $\overline{E K E}$. The Agulhas Return Current $\overline{E K E}$ has moderate values $\left(O(2-3) \times 10^{3} \mathrm{~cm}^{2} \mathrm{~s}^{-2}\right)$ reflecting the two standing meanders found at the Agulhas Plateau $\left(26^{\circ} \mathrm{E}\right)$ and at $32^{\circ} \mathrm{E}$. The observed and the modeled $\overline{E K E}$ magnitudes differ upstream of $23^{\circ} \mathrm{E}$. The difference is the largest at the Agulhas Bank eastern margin $\left(25^{\circ} \mathrm{E}-23^{\circ} \mathrm{E}\right)$. The model shows an intense $\overline{E K E}$, with values of the same order as at the Retroflection area, whereas AVISO shows a lower magnitude $\left(O(1.5) \times 10^{3} \mathrm{~cm}^{2} \mathrm{~s}^{-2}\right)$. This area is where Natal Pulses can be trapped and grow in size (Krug et al., 2014) and where Shear-Edge eddies can develop (Lutjeharms et al., 1989) and potentially leak downstream. The amplitude difference between the two datasets can be explained by their different spatial resolutions. AVISO spatial effective resolution is $\lambda \sim 100-150 \mathrm{~km}$ (Chelton et al., 2011) whereas the $\mathrm{dx} \sim 2.5 \mathrm{~km}$ nest has an effective resolution of about $\lambda \sim 10 d x \sim 25 \mathrm{~km}$ (Soufflet et al., 2016). Hence, scales in the range of 10-100 km seem to drive the Agulhas Current variability at the Agulhas Bank eastern margin. The relationship between the relaxation of the topographic constraint on the current and the development of intense perturbations (Lutjeharms, 2006; Paldor \& Lutjeharms, 2009) is visible in the modeled $\overline{E K E}$ (Figure 4b). Areas of intense $\overline{E K E}$ are associated with the current path (sea surface height contours in green) separating from the topography (1000 $\mathrm{m}$ isobath is the most inshore black contour).

\subsection{The Agulhas Current Upper Branch (Upstream of Port Elizabeth): Description of the Inshore} Front

3.2.1. Mean Vertical Structure of the Agulhas Current at 28.5 E: Comparison With ACT Data

The Agulhas Current mean vertical structure from the 1 year-long simulation of the highest-resolution nest ( $\mathrm{dx} \sim 0.75 \mathrm{~km}$ ) is compared to the Agulhas Current Time-series Experiment (ACT) data (Figure 5). ACT is a 34 month-long time series (April 2010-February 2013) of current measurements undertaken from a mooring array across the Agulhas Current at $28.5^{\circ} \mathrm{E}$ (Beal et al., 2015). 

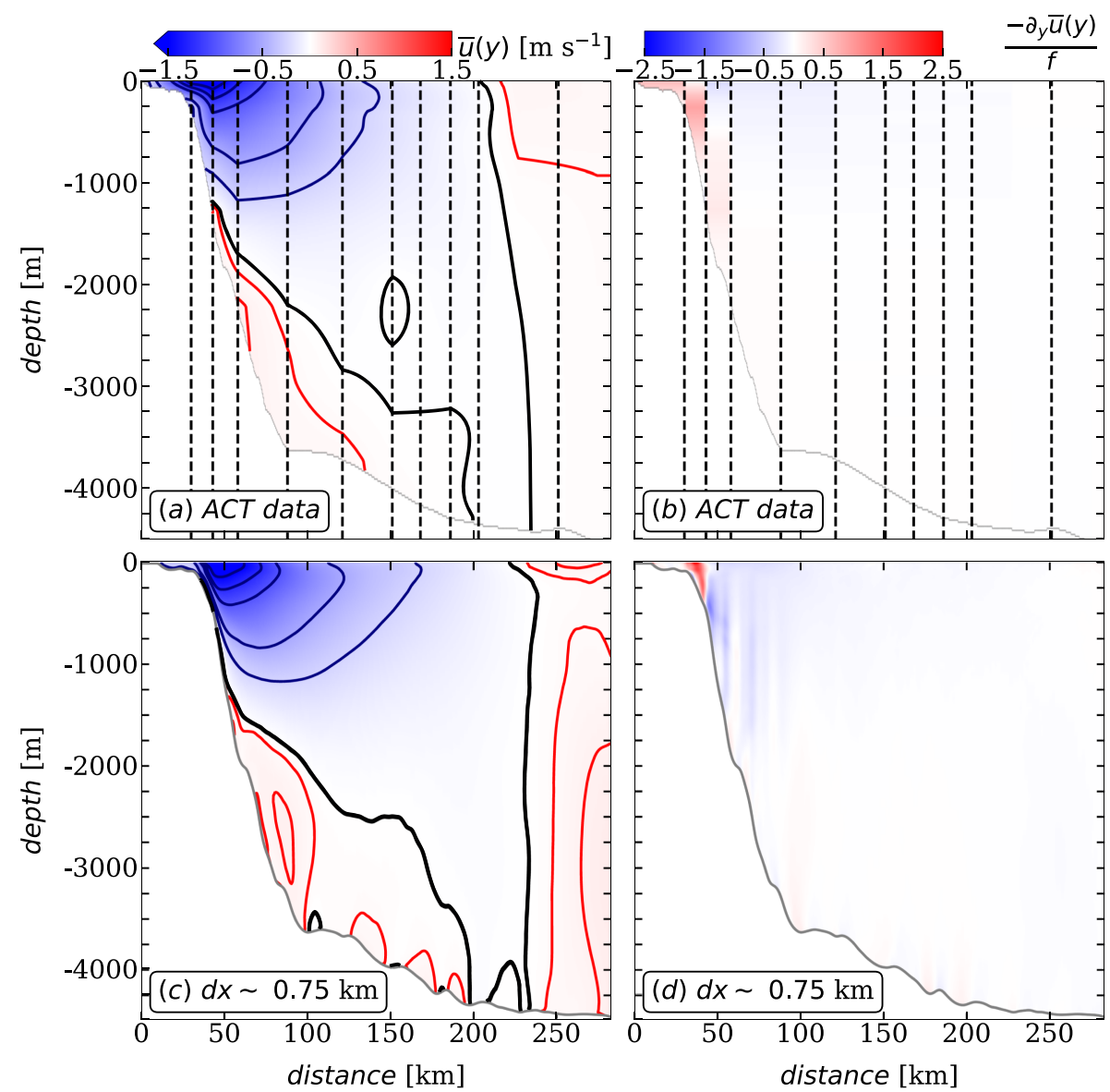

Figure 5. Sections of the mean along-front velocity $\left(\bar{u}(y)\left[\mathrm{m} \mathrm{s}^{-1}\right]\right)$ and of the mean normalized relative vorticity $-\frac{\partial_{y} \bar{u}(y)}{f}$ around $28.5^{\circ} \mathrm{E}$ from the ACT data set (a and b) and from the model at $\mathrm{dx} \sim 0.75 \mathrm{~km}$ (c and d). The location of the ACT array is shown by the black segment in Figure $4 \mathrm{~b}$. The black dashed lines locate the seven full-depths current meter moorings. On the mean along-front velocity panels, the blue contours are the $-0.25,-0.5,-1,-1.25$, and $-1.5 \mathrm{~m} \mathrm{~s}^{-1}$ isolines, the red contours are the 0.03 and $0.08 \mathrm{~m} \mathrm{~s}^{-1}$ isolines and the bold black contour is the $0 \mathrm{~m} \mathrm{~s}^{-1}$ isoline. The observed and modeled mean vertical structures of the Agulhas Current show an intense cyclonic front expanding from the surface to $500 \mathrm{~m}$.

The modeled Agulhas Current has a vertical structure close to the one observed during the ACT experiment. They both have a similar width of $200 \mathrm{~km}$, defined as the distance between the $0 \mathrm{~m} \mathrm{~s}^{-1}$ velocity contour (Figures $5 \mathrm{a}$ and $5 \mathrm{c}$ ), in the upper ocean. In the model, the current extends slightly further offshore than in the observations and does not intrude significantly onto the continental shelf. This results in a clear delimitation between the shelf waters and the Agulhas Current. The modeled and observed currents have a similar vertical structure, with a v-shape typically associated with surface intensified flows. The mean Agulhas Current velocities reach values larger than $-1.5 \mathrm{~m} \mathrm{~s}^{-1}$ at the surface and the current core, defined by the $-0.5 \mathrm{~m} \mathrm{~s}^{-1}$ contour, extends over a width of about $100 \mathrm{~km}$ at the surface and is $800 \mathrm{~m}$ deep. These similarities in the mean Agulhas structure are reflected in the net volume flux ( $T_{b o x}$ in Beal et al., 2015): $-77 \pm 5 \mathrm{~Sv}$, for the ACT data and $-79.6 \mathrm{~Sv}$ for the highest-resolution nest.

The observed and modeled Agulhas inshore fronts have high values of cyclonic relative vorticity which is uniform from the surface to about $500 \mathrm{~m}$ depth (Figures $5 \mathrm{~b}$ and $5 \mathrm{~d}$ ). The modeled front is more intense with relative vorticity values up to $O(3 \mathrm{f})$ compared to the observations of $O(0.5 \mathrm{f})$. This magnitude difference could suggest different mean current structures. The modeled current does not spread onto the continental shelf and this results in a sharper front at the shelf break. However, the coarser horizontal resolution of the ACT data, which cannot resolve the sharp current gradients at the inshore front of the Agulhas Current, must also contribute to this difference. 

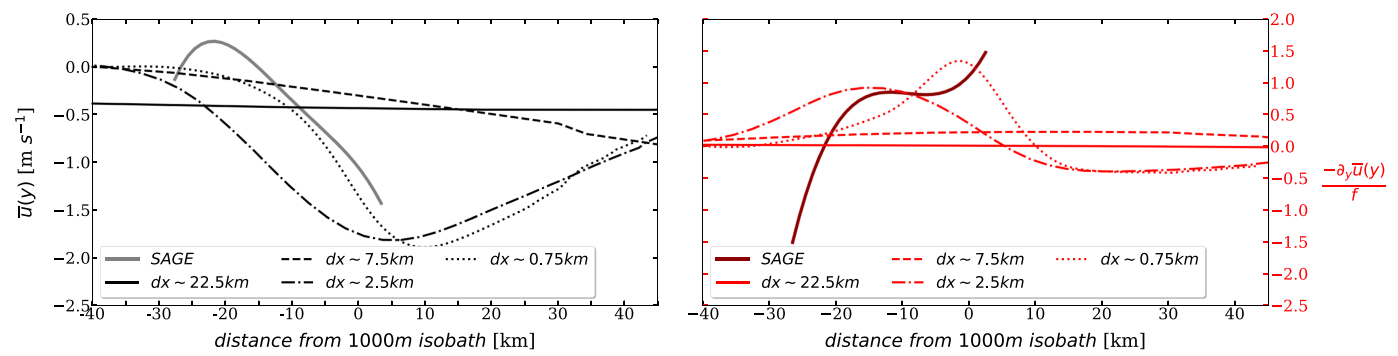

Figure 6. Cross-front sections of the mean along-front velocities $\left(\bar{u}(y)\left[\mathrm{m} \mathrm{s}^{-1}\right]\right.$, left panel) and relative vorticity $-\frac{\partial_{y} \bar{u}(y)}{f}$, right panel at the SAGE location $\left(25.75^{\circ}\right.$ E, shown in Figure 2a) for the SAGE glider data (Krug et al., 2017; gray and dark red lines) and for the four model nests at $\mathrm{dx} \sim 22.5$ (plain lines), 7.5 (dashed-dotted lines), 2.5 (dashed lines), and $0.75 \mathrm{~km}$ (dotted lines) resolution. The bottom x-axis represents the cross-front distance from the $1000 \mathrm{~m}$ isobath, which represents the front position for the SAGE period. The highest-resolution nest has a frontal structure close to the SAGE dataset.

The agreement between the highest-resolution nest and the ACT data also include the Agulhas Undercurrent mean vertical structure. Below the Agulhas Current flows a weaker equatorward current: the Agulhas Undercurrent (Figures 5a and 5c). Both observed and modeled deep currents show a vein of weak northeasterly flow between 1500 and $3000 \mathrm{~m}$ over a width of $50 \mathrm{~km}$. This undercurrent embraces the topography and is associated with mean velocities of about $0.03 \mathrm{~m} \mathrm{~s}^{-1}$ and peaking up to $0.08 \mathrm{~m} \mathrm{~s}^{-1}$ where it is the closest to the topography.

\subsubsection{Mean Surface Structure of the Agulhas Cyclonic Front at $25.75^{\circ}$ E: Comparison With SAGE Data}

The Agulhas Current mean surface structure, along the SAGE section $\left(25.75^{\circ} \mathrm{E}\right)$, from the four model nests $(\mathrm{dx} \sim 22.5,7.5,2.5$, and $0.75 \mathrm{~km}$ ) are compared to glider data from SAGE (Figure 6). With the SAGE dataset, the cross-front section is computed by averaging the data in the along-front direction and over the 1 month-long record. The model sections are computed following the same steps for a period equivalent to the SAGE one. In the model, the 1-month period is chosen in order to get similar dynamical conditions as during the SAGE experiment regardless of the dates. This 1-month period corresponds to a period without mesoscale meanders, that is, a nonmeandering state, the $1000 \mathrm{~m}$ isobath marks the mean inshore front position (not shown here) and submesoscale eddies are present. Contrary to the SAGE period, no northeastward current on the shelf is present during the 1-month period in the model. The origin of this feature on the shelf is unclear (Krug et al., 2017), and it is shown in the next sections that it is not a limiting factor for our study.

The highest-resolution nest $(\mathrm{dx} \sim 0.75 \mathrm{~km})$ best fits with SAGE observations. The modeled and observed along-front velocities have close cross-front patterns and magnitude offshore of the shelf (about $-1 \mathrm{~m} \mathrm{~s}^{-1}$ at the inshore front location). The modeled vorticity shows a pattern typical of sheared flows, with a peak of $O(2 \mathrm{f})$ at the front location, whereas the observed vorticity reaches a threshold at the front $(O(1 \mathrm{f}))$ and increases offshore. The limited number of observations collected during SAGE could explain why the modeled and observed relative vorticity cross-front profiles differ. The gliders did not go further offshore to catch the maximum magnitude of the current and hence cannot show a decrease after reaching a relative vorticity maximum.

All the modeled cross-front sections, relative to different numerical resolutions, show different patterns. This suggests that the highest-resolution nest did not converge yet. While the $\mathrm{dx} \sim 22.5$ and $7.5 \mathrm{~km}$ nests have flat cross-front patterns, the $\mathrm{dx} \sim 2.5$ and $0.75 \mathrm{~km}$ nests show gradients of mean along-front velocity and mean vorticity peaking at the mean Agulhas inshore front position (1000 m isobath). The lack of convergence means the highest-resolution nest could be numerical resolution-dependent. This could also explain the different relative vorticity cross-front patterns between the model and SAGE. However, in the next sections it is shown that in spite of the lack of convergence, the highest-resolution nest and SAGE captured the same frontal dynamics.

In this section, we show that the model captures the mesoscale variability of the Agulhas Current (Figure 4). Perturbations are the most energetic along the downstream branch of the current: at the Agulhas Bank, at the Retroflection, and along the Agulhas Return Current. In the vicinity of Port Elizabeth $\left(26^{\circ} \mathrm{E}\right)$, the model 

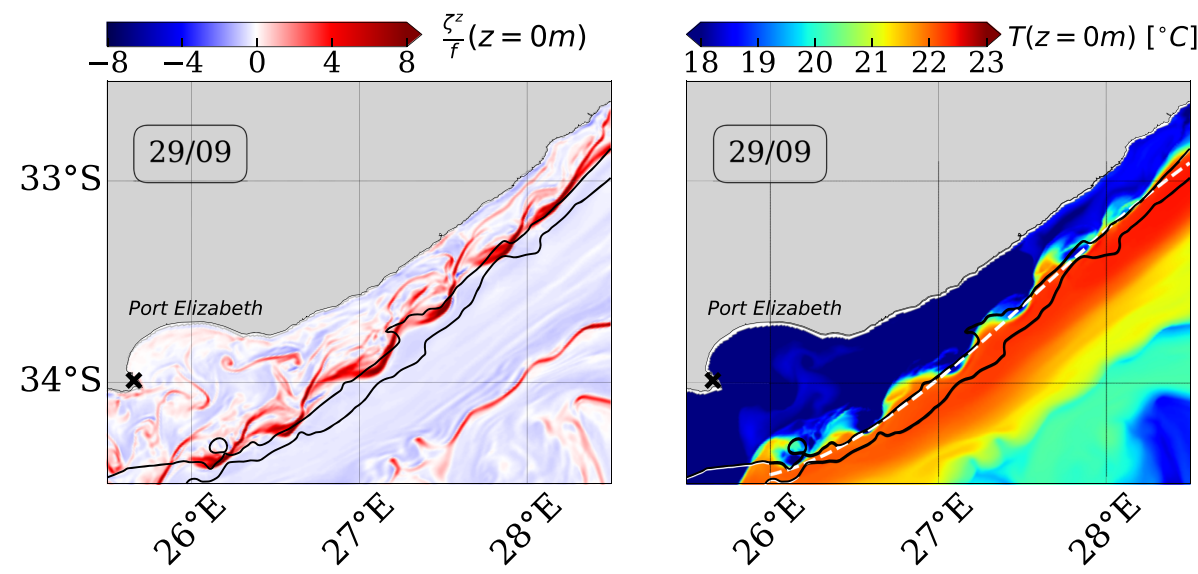

Figure 7. Snapshots of the surface normalized vertical relative vorticity $\frac{\zeta^{z}}{f}$, left panel and of the temperature $\left(\mathrm{T}\left[{ }^{\circ} \mathrm{C}\right]\right.$, right panel) when submesoscale eddies are present. Black contours are the 200 and $1000 \mathrm{~m}$ isobaths. The white dashed line represents the location of the vertical sections plotted along the vortex street in Figures 8 and 10 bottom panel. The vortex street develops along the front between $28^{\circ} \mathrm{E}$ and $26^{\circ} \mathrm{E}$.

shows, in good agreement with in situ data, that the mean Agulhas inshore front has an intense cyclonic relative vorticity $(O(>f))$ that is uniform from the surface to about $500 \mathrm{~m}$ depth (Figure 5). Despite the respective limitations of the model and SAGE data - the model might not have converged yet and the glider data are sampled over a limited area - the modeled mean-state is similar to the SAGE one (Figure 6). We also validated the modeled water masses at the ACT array against the CARS climatology (Ridgway et al., 2002) (not shown here).

\section{Submesocale Eddies along the Agulhas Cyclonic Front}

In this section, we describe the submesoscale frontal eddies in the model, we investigate barotropic instability as the generation mechanism proposed by Krug et al. (2017), and we evaluate its importance in driving the Agulhas inshore front variability.

\subsection{Description of the Submesoscale Cyclonic Eddies in the SAGE Area}

Submesoscale eddies (diameters of 10-20 km) develop along the Agulhas inshore front upstream of Port Elizabeth $\left(26^{\circ} \mathrm{E}\right)$ in the highest-resolution nest (Figure 7). Several eddies develop simultaneously between $28^{\circ} \mathrm{E}$ and $26^{\circ} \mathrm{E}$, over a portion of the front where the current flows closely to the topography, and forms a vortex street. In the model, submesoscale eddies are generated on a quasi-regular basis. They have a limited life-time ( $\sim 3-4$ days). Once developed, they propagate downstream, in the opposite direction of Kelvin waves, over 300-400 km before dissipating. Along the Agulhas Bank (westward of Port Elizabeth, $26^{\circ} \mathrm{E}$ ), the topographic constraint on the front relaxes. As a result, the submesoscale eddies can grow to diameters of $40-50 \mathrm{~km}$, beyond the submesoscale range.

The size and location of the modeled submesoscale eddies are consistent with the submesoscale eddies captured during SAGE (Krug et al., 2017) and observed on SST satellite images (Figure 2).

The submesoscale eddies are intensified at the surface and associated with a cold temperature anomaly (Figure 8). With values of $(O(5-10) f)$, the relative vorticity is the largest in the eddy cores and within the upper $25 \mathrm{~m}$ (Figure 8 top panel). Their core temperatures range from 15 to $19^{\circ} \mathrm{C}$, and at the edges, it ranges from 20 to $23^{\circ} \mathrm{C}$ (Figure 8 bottom panel). The cold temperature anomaly is related to the cyclonic motion of the eddies which induces an upward doming of the isotherms. Water mass horizontal transfer during the process of the front destabilization can also reinforce the eddy temperature anomaly. In fact, the Agulhas front, characterized by warm water, develops cross-frontal meanders which extend onto the shelf and trap local cold water when they grow into finite amplitude and form the cyclonic eddies.

\subsection{Barotropic Instability as the Generation Mechanism}

The Agulhas inshore front is barotropically unstable before the submesoscale eddy generation. The submesoscale eddies develop along a current portion where the mean velocity shear is intense (Figures 5 and 6) and such flows are prone to barotropic instability. A necessary condition for this instability is a change of sign of 

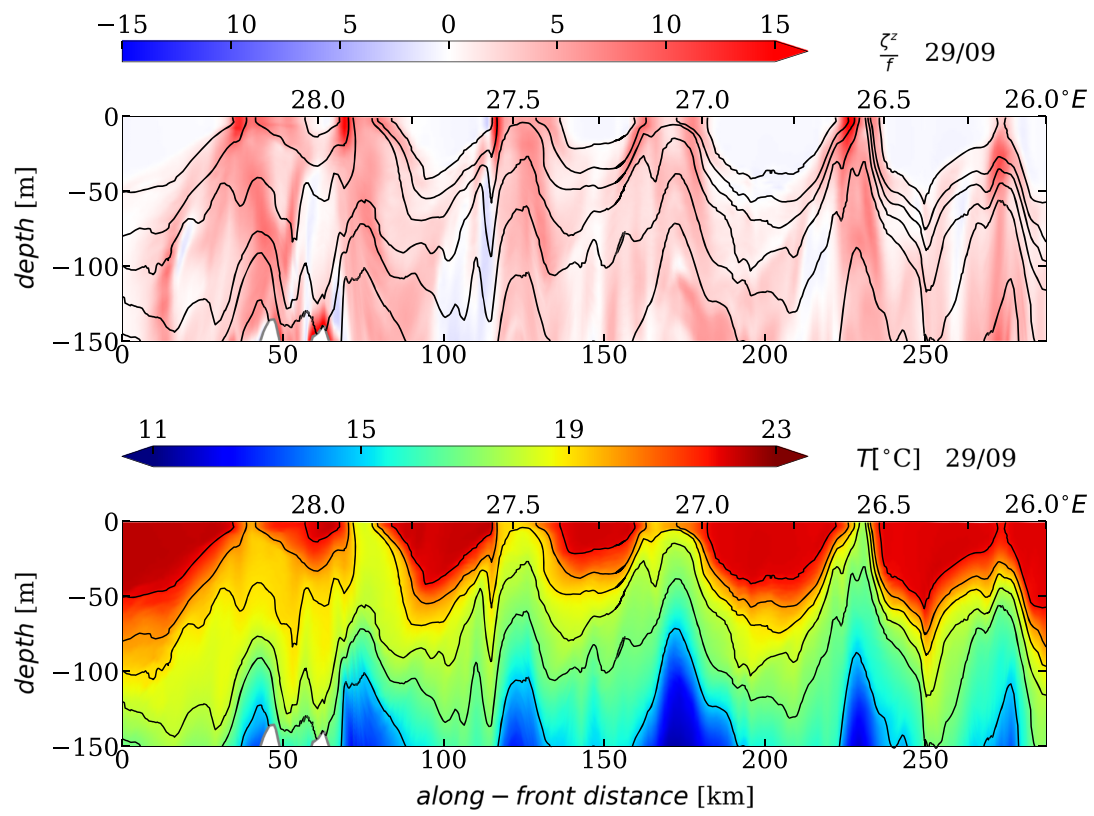

Figure 8. Sections along the vortex street, between $28.5^{\circ} \mathrm{E}$ and $26^{\circ} \mathrm{E}$, plotted at the same time than in Figure 7. The sections show the normalized relative vorticity $\frac{\zeta^{z}}{f}$, top panel and the temperature ( $\left.{ }^{\circ} \mathrm{C}\right]$, bottom panel). The black contours are the $15,17,18,19,20$, and $21.75^{\circ} \mathrm{C}$ isotherms. The location of the sections along the vortex street is shown as the white dashed line in Figure 7. On the vertical, the submesoscale cyclonic eddies are surface-intensified with cold-cores and large relative vorticity values.

$\frac{\partial^{2} U(y)}{\partial y^{2}}$, with $y$ the cross-front direction and $U(y)$ the along-front velocity component. A typical velocity profile $U(y)$, selected from the highest-resolution nest below the surface $(5 \mathrm{~m})$ upstream of the eddy generation site $\left(28^{\circ} \mathrm{E}\right)$ and at a time just before eddies develop (Figure 9 top panel), satisfies the instability condition.

Perturbations originating from the barotropically unstable velocity profile are coherent with the submesoscale eddies. A linear stability analysis is performed with the modeled velocity profile shown in (Figure 9 top panel). The Boussinesq eigenvalue problem described by Ménesguen et al. (2012) is modified to a one-dimensional horizontal problem. The linear stability problem is solved for a typical velocity state $U(y)$ and normal modes for the perturbations $\{u(y), v(y), p(y)\} e^{i(m y)} e^{\sigma t}$, with $y$ the cross-front direction, $u$ and $v$ the along and cross-front velocity components, $p$ the pressure, $m$ the nondimensional $\mathrm{x}$-wavenumber, one-dimensional the adimensionalized Boussinesq equations for momentum are

$$
\begin{aligned}
R o[\sigma v+i m U v]-u+\partial_{y} p-E k \Delta^{\prime} v & =0 \\
R o\left[\sigma u+\partial_{y} U v-i m U u\right]+v+i m p-E k \Delta^{\prime} u & =0 \\
\partial_{y} v+i m u & =0
\end{aligned}
$$

with $\Delta^{\prime}=\partial_{x^{2}}^{2}-m^{2}$.

The initial velocity profile $U(y)$ corresponds to the function: $U(y)=U_{0} \tanh \left(\frac{y}{d}\right)+U_{1}$ fitted to the velocity profile from the highest-resolution nest (Figure 9 top panel), with $U_{0}=-1.09 \mathrm{~m} \mathrm{~s}^{-1}$ the typical velocity magnitude, $d=830 \mathrm{~m}$ the shear width and $U_{1}=-0.32 \mathrm{~m} \mathrm{~s}^{-1}$ a constant. For the system of equation (1), the one-direction flow is governed by two nondimensional numbers: the Rossby and the Ekman numbers. The Rossby number $R o=\frac{U_{0}}{f d}$, with $f$ the Coriolis parameter, is the ratio of inertial to rotation terms and is as large as $R o=16$. The Ekman number $E k=\frac{v}{f d^{2}}$, with $v$ the viscosity, is the ratio of diffusion to rotation terms and is kept very small $\left(10^{-6}\right)$ to correspond to inviscid flow.

The fastest growing modes have wavelengths of 10-13 km (Figure 9 bottom panel). The one-dimensional linear stability analysis points out perturbations in the same scale range as the submesoscale eddies in the highest-resolution nest (10-20 km, Figure 7) and as the ones captured by SAGE and observed on the SST satellite images ( $15 \mathrm{~km}$, Figure 2). 

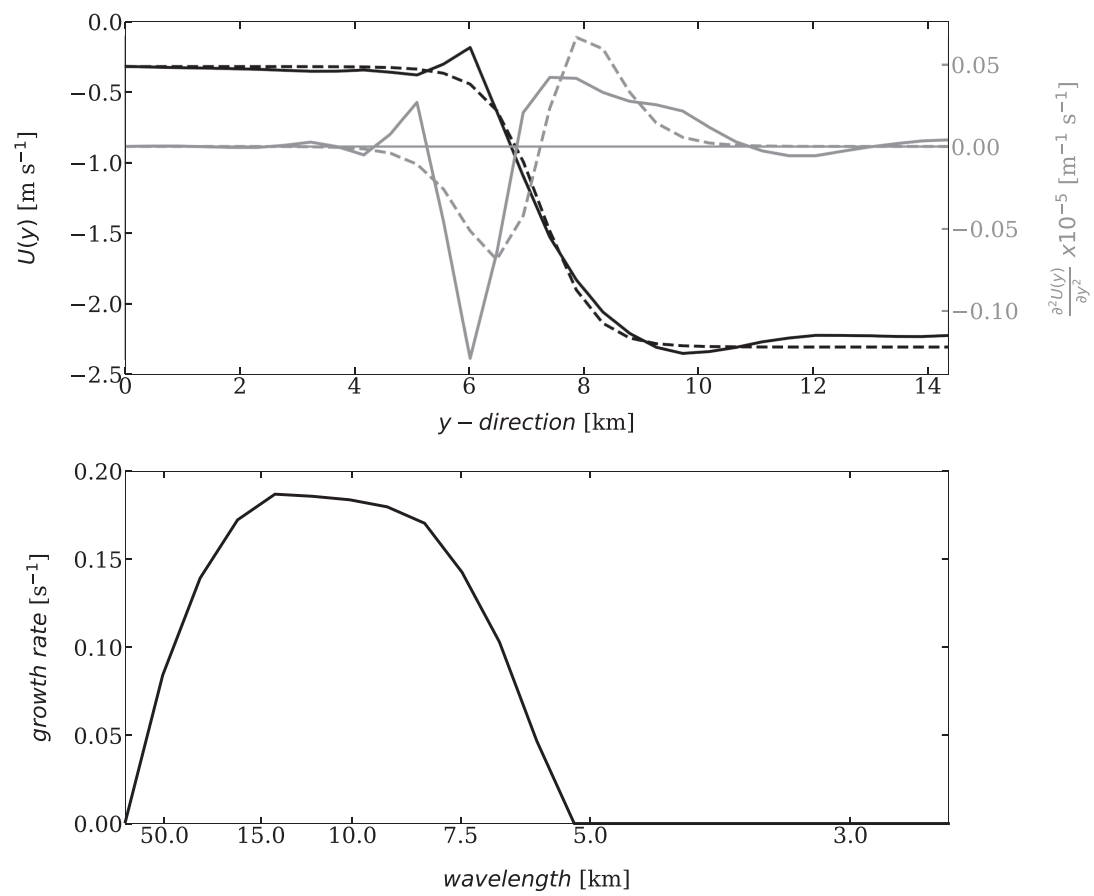

Figure 9. The cross-front velocity profile $\left(U(y)\left[\mathrm{m} \mathrm{s}^{-1}\right]\right.$ in plain black line) and its fit (dashed black line) used as initial conditions in the one-dimensional linear stability analysis are plotted (top). It is picked from the model at $5 \mathrm{~m}$ depth, at $28^{\circ} \mathrm{E}$, just upstream of the submesoscale eddies generation, for a snapshot just before the submesoscale eddies develop. The relative vorticity gradient, $\frac{\partial^{2} U(y)}{\partial y^{2}}\left[\mathrm{~m}^{-1} \mathrm{~s}^{-1}\right]$, computed for the initial velocity profile (plain gray line) and its fit (dashed gray line) are also plotted. The cross-front velocity profile satisfies the barotropic instability necessary condition. The perturbation growth rate $\left[\mathrm{s}^{-1}\right]$, resulting from the one-dimensional linear stability analysis, is plotted as a function of wavelength $[\mathrm{km}]$ (bottom). The one-dimensional linear stability analysis shows perturbations at the same scale $(15 \mathrm{~km})$ than the submesoscale eddies seen in the model $(10-20 \mathrm{~km})$ and in the SAGE data set $(15 \mathrm{~km})$.

The parameter $d$ corresponds to the shear width and it is close to the numerical resolution: $\mathrm{dx} \sim 0.75 \mathrm{~km}$. This is consistent with the possible nonconvergence of the highest-resolution nest, as hinted by Figure 6 , and this means that the mean velocity shear amplitude could be limited by the model viscosity. However, this is also counterbalanced by the modeled shear being of the same order of magnitude as the shear measured during SAGE (Figure 6).

This preliminary analysis, led for a specific time of submesoscale eddy generation, supports Krug et al.'s (2017) suggestion that barotropic instability is the main generation mechanism.

The eddy kinetic energy source, identified for the same episode of submesoscale eddy generation, confirms the prevalence of barotropic instability. The $\overline{E K E}$ equation is (Gula et al., 2016b):

$$
\begin{aligned}
& \underbrace{\frac{1}{\frac{\partial u_{i}^{\prime 2}}{\partial t}}}_{\frac{\partial E K E_{i}}{\partial t}}+\underbrace{\frac{\partial}{\partial x_{i}}\left(\frac{1}{2} \overline{u_{j}} \overline{u_{i}^{\prime 2}}+\frac{1}{2} \overline{u_{j}^{\prime} u_{i}^{\prime 2}}+\frac{1}{\rho_{0}} \overline{u_{j}^{\prime} p^{\prime}}\right)}_{\text {Boundary }}= \\
& \underbrace{-\overline{u_{j}^{\prime} u_{i}^{\prime}} \frac{\partial \overline{u_{i}}}{\partial x_{j}}}_{M K E \rightarrow \overline{E K E}}+\underbrace{\overline{w^{\prime} b^{\prime}}}_{\overline{E P E} \rightarrow \overline{E K E}}+\underbrace{\overline{V_{i}^{\prime} u_{i}^{\prime}}}_{\text {Vertical mixing }}+\underbrace{\overline{D_{i}^{\prime} u_{i}^{\prime}}}_{\text {Horizontal }} \\
& \text { with } M K E \rightarrow \overline{E K E}=\overbrace{-\overline{u_{i}^{\prime} u_{i}^{\prime}} \frac{\partial \overline{u_{i}}}{\partial x_{i}}}^{\overline{H R S}}-\overbrace{\overline{u_{i}^{\prime} w^{\prime}} \frac{\partial \overline{u_{i}}}{\partial z}}^{\overline{V R S}} \text { and } \overline{E P E} \rightarrow \overline{E K E}=\overbrace{\overline{w^{\prime} b^{\prime}}}^{\overline{V B F}}
\end{aligned}
$$

The Cartesian tensor notation with summation convention is used with $i=1,2$ and $j=1,2,3 . u_{i}$ are the horizontal velocity components, $u_{3}=w$ is the vertical velocity, $p$ is the pressure anomaly, $b=\frac{-g \rho}{\rho_{0}}$ is the buoyancy 

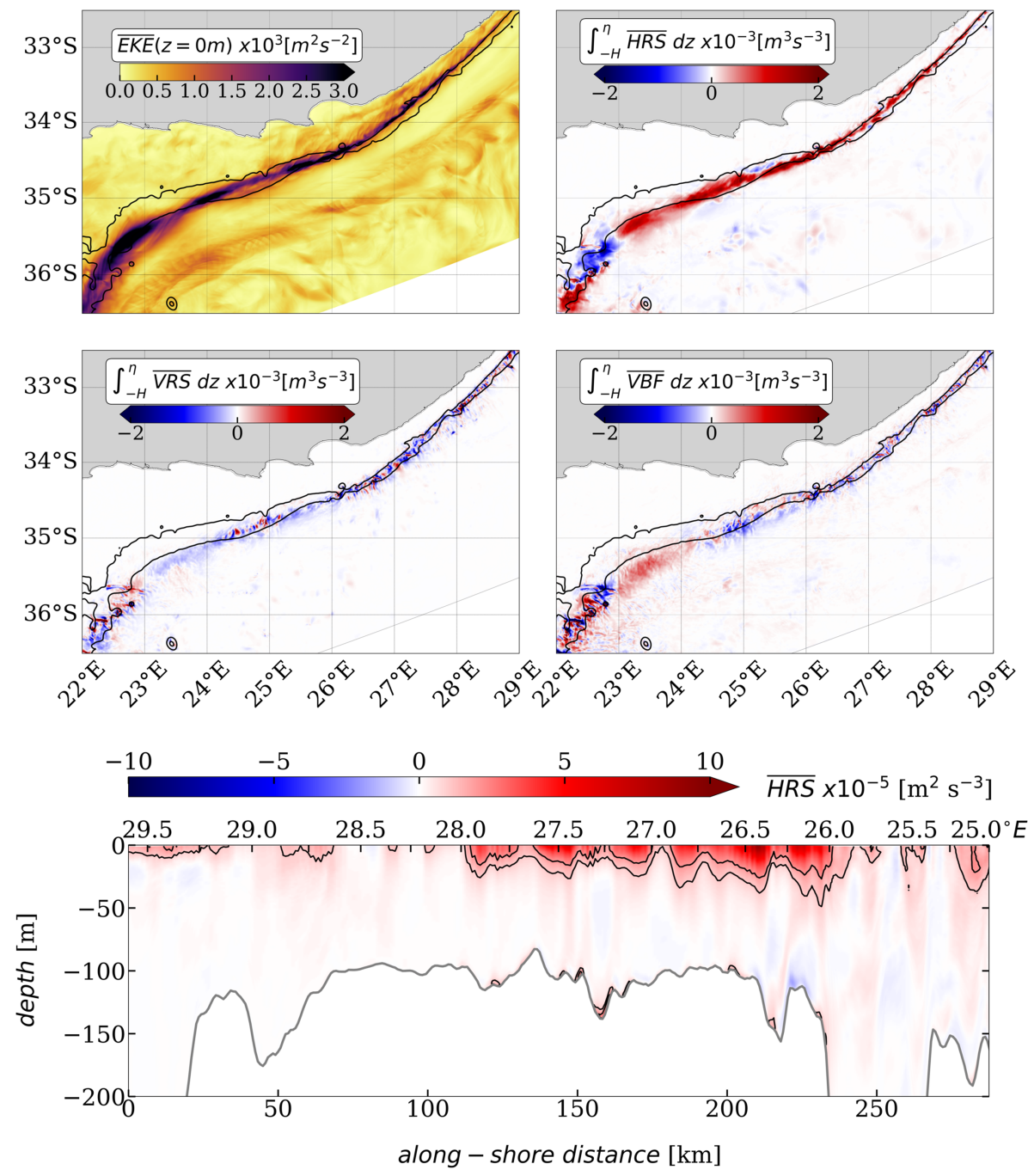

Figure 10. Top four panels : Maps of surface $\overline{E K E}\left[\mathrm{~cm}^{2} \mathrm{~s}^{-2}\right]$ and energy conversion rates $\overline{H R S}, \overline{V R S}$, and $\overline{V B F}\left[\mathrm{~m}^{-3} \mathrm{~s}^{-3}\right]$ integrated over the entire water column. The overbar denotes a week-long average. This average encompasses the Figure 7 snapshot (09/29 06:00) and corresponds to a full event of submesoscale eddies generation. Black contours represent the 200 and $1000 \mathrm{~m}$ isobaths. When submesoscale eddies are presents, $\overline{H R S}$ is the only term accounting for the energy transfer $M K E \rightarrow \overline{E K E}$. Bottom panel: Section along the vortex street, between $29.5^{\circ} \mathrm{E}$ and $25^{\circ} \mathrm{E}$, of the energy conversion rate $\overline{H R S}\left[\mathrm{~m}^{2} \mathrm{~s}^{-3}\right]$. The overbar denotes the same time average than in the top four panels. Black contours follow the 1 and $2 \mathrm{~m}^{2} \mathrm{~s}^{-3} \overline{H R S}$ isolines. The location of the section along the vortex street is shown as the white dashed line in Figure 7. The energy transfer $M K E \rightarrow \overline{E K E}$ occurs between the surface and $50 \mathrm{~m}$ deep.

anomaly, $V_{i}^{\prime}$ is the vertical mixing, with $V_{i}^{\prime}=\frac{\partial}{\partial_{z}}\left(K_{M v}\left(\frac{\partial u_{i}^{\prime}}{\partial_{z}}\right)\right)$ and $K_{M v}$ the momentum eddy diffusivity, and $D_{i}^{\prime}$ is the horizontal diffusion term in the horizontal momentum equations, computed as the difference between the third-order upwind numerical advective momentum scheme and the fourth-order centered scheme (Gula et al., 2014). The overbar denotes a time mean and the prime denotes fluctuations relative to this mean. In equation (2), the terms from the left hand side correspond to the Eulerian time derivative and to the boundary transport of $\overline{E K E}$, due to pressure fluctuations and advection by the mean and fluctuative current components, whereas the terms from the right hand side correspond to the local sources and sinks. The two terms $M K E \rightarrow \overline{E K E}$ and $\overline{E P E} \rightarrow \overline{E K E}$ are possible $\overline{E K E}$ sources while vertical mixing and lateral diffusion are the main sinks. The barotropic instability signature on energy transfer is a positive $M K E \rightarrow \overline{E K E}$ transfer through its horizontal component: $\overline{H R S}$. It corresponds to an energy extraction from the mean horizontal shear toward the growing perturbations.

The three possible $\overline{E K E}$ sources $(\overline{H R S}, \overline{V R S}$ and $\overline{V B F})$ are averaged over a 1-week period which corresponds to the full period of the submesoscale eddy generation event previously investigated (Figure 10). Along the 


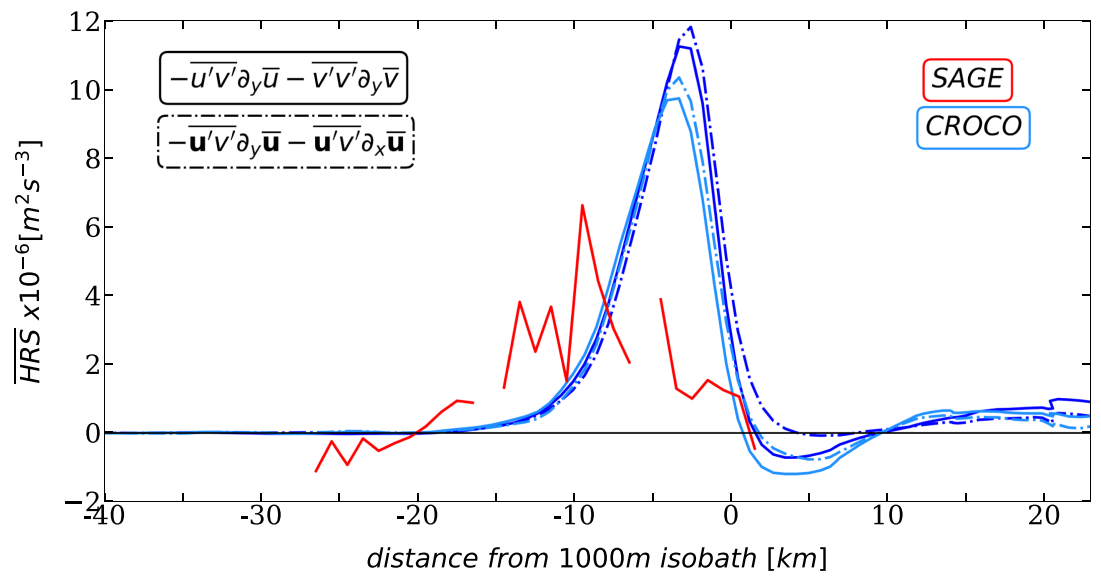

Figure 11. Cross-front sections at the SAGE location $\left(25.75^{\circ}\right.$ E, shown in Figure 2a) for the surface observed (red) and modeled (blue) partial and full $\overline{H R S}$ rate. The partial $\overline{H R S}$ rate, only composed of the cross-front derivatives subterms $\left(-\overline{u^{\prime} v^{\prime}} \partial_{y} \bar{u}-\overline{v^{\prime} v^{\prime}} \partial_{y} \bar{v}\right)$, is shown for the SAGE data set as a plain red line and for the model in plain blue lines. The full $\overline{H R S}$ rate, composed of the cross and along-front derivatives subterms $\left(-\overline{u^{\prime} \boldsymbol{u}^{\prime}} \partial_{x} \overline{\boldsymbol{u}}-\overline{v^{\prime} \boldsymbol{u}^{\prime}} \partial_{y} \overline{\boldsymbol{u}}\right)$ is shown in dashed blue lines. The modeled partial and full $\overline{H R S}$ rates are computed for different time periods : a 1-month long (light blue lines) and five consecutive months without Natal Pulses (dark blue lines). The bottom x-axis represents the cross-front distance from the $1000 \mathrm{~m}$ isobath, which represents the front position for the SAGE period. The comparison between the modeled partial and full $\overline{H R S}$ rates shows that the cross-front derivatives are a good proxy of the full $\overline{H R S}$ rate. The comparison between the modeled 1- and 5-month averages of the $\overline{H R S}$ rates shows that the submesoscale eddies generation is a locally recurrent process.

front portion, where the submesoscale eddies develop $\left(28^{\circ} \mathrm{E}-26^{\circ} \mathrm{E}\right), \overline{H R S}$ is positive and has the highest magnitude whereas $\overline{V R S}$ and $\overline{V B F}$ are lower and mainly negative. $\overline{H R S}$ is here the dominant energy source term for the perturbations $(M K E \rightarrow \overline{E K E}$ ). This confirms the role of barotropic instability for the generation of submesoscale eddies along the Agulhas Current inshore front. The instability process is thus very similar to the development of barotropic instabilities in the submesoscale range described for the Gulf Stream (Gula et al., 2015b).

The vertical structure of the energy conversion term $\overline{H R S}$ (Figure 10 bottom panel) matches the surface-intensified structure of the submesoscale eddies (Figure 8). Indeed, along the vortex street $\left(29.5^{\circ}\right.$ $\mathrm{E}-25^{\circ} \mathrm{E}$ ) the energy conversion term is intensified from the surface to a depth of about $50 \mathrm{~m}$.

In this subsection, we focus on a specific event of submesoscale eddy generation (Figures 7 and 8 ). In the next subsection, we show that the generation of submesoscale eddies by barotropic instability is recurrent in this region, and we discuss its predominance in driving the local variability of the Agulhas inshore front.

\subsection{Barotropic Instability as a Locally Preponderant Mechanism}

The triggering of the barotropic instability along the Agulhas inshore front is compared between the model and the SAGE data. With the 1-month long glider dataset, a partial $\overline{H R S}$ term can be computed along a cross-front section at $25.75^{\circ} \mathrm{E}$ (Krug et al., 2017). This partial term accounts for the cross-front derivative subterms: $-\overline{u^{\prime} v^{\prime}} \partial_{y} \bar{u}-\overline{v^{\prime} v^{\prime}} \partial_{y} \bar{v}$, the overbar denotes a monthly time average, which corresponds to the full SAGE period, and the prime denote fluctuations relative to this time mean. This partial $\overline{H R S}$ term is positive and reaches a maximum at the Agulhas front. This result should be considered with caution since it involves several approximations on spatial and time averages, and their findings might be limited by their use of a partial $\overline{H R S}$.

The modeled partial $\overline{H R S}$ term, computed over an equivalent period to the SAGE one (presented in section 3.2.2, Figure 6), is compared to the SAGE one along the SAGE section (25.75 E; Figure 11$)$. The modeled (blue lines) and the observed (red line) $\overline{H R S}$ have similar patterns. They reach a maximum energy conversion rate inshore the $1000 \mathrm{~m}$ isobath, corresponding to the mean front location, with close magnitudes : 6 $\times 10^{-6}$ and $1.08 \times 10^{-5} \mathrm{~m}^{2} \mathrm{~s}^{-3}$, respectively. These maximum values are also in agreement with those found in the Gulf Stream $\left(1 \times 10^{-5} \mathrm{~m}^{2} \mathrm{~s}^{-3}\right)$ by Gula et al. (2015b). Hence, the approximations on spatial and time averages required by the SAGE data for the $\overline{H R S}$ computation do not create a strong bias in the energy conversion term. Similarly, the presence of a northeastward current on the shelf during the SAGE experiment 


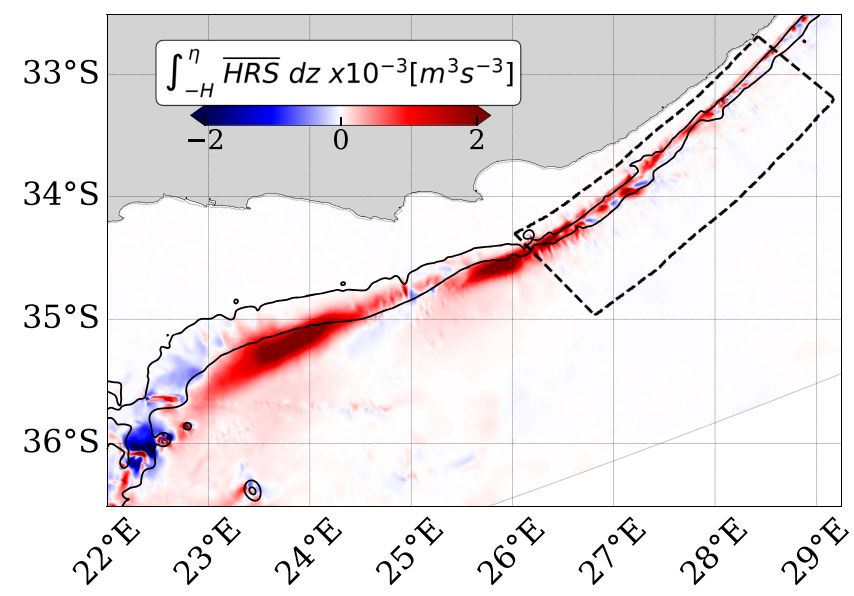

Figure 12. Map of energy conversion rate $\overline{H R S}\left[\mathrm{~m}^{3} \mathrm{~s}^{-3}\right]$, integrated in the vertical. The overbar denotes a 5 month-long time average, when no Natal Pulse is present. Black contours are the 200 and $1000 \mathrm{~m}$ isobaths. The black dashed box represents the area used for the computations of the along-front distributions (Figures 13) and of the time series (Figure 15). The 1-week (Figure 6) and 5 month-long time averages of $\overline{H R S}$ show a similar pattern and confirm the hypothesis that submesoscale eddy generation is a locally recurrent process.

(Figure 6), which is absent in the model, does not create discrepancies relevant for the Agulhas inshore frontal dynamics.

The energy transfer $M K E \rightarrow \overline{E K E}$ realized by the $\overline{H R S}$ term is mainly attributable to the mean current lateral shear : $\overline{u^{\prime} v^{\prime}} \partial_{y} \bar{u}$. In the model, the partial and the full versions of the $\overline{H R S}$ term $\overline{-u^{\prime} \boldsymbol{u}^{\prime}} \partial_{x} \overline{\boldsymbol{u}}-\overline{v^{\prime} \boldsymbol{u}^{\prime}} \partial_{y} \overline{\boldsymbol{u}}$, with $\boldsymbol{u}$ the vector of the horizontal velocity components, are compared. Both terms show the same patterns with only small magnitude differences. Since the upstream Agulhas Current is a coastal jet constrained by a steep and straight topography, its frontal structure is dominated by the cross-shelf structure. Hence, Krug et al.'s (2017) estimation of the energy conversion at the Agulhas inshore front using a partial $\overline{H R S}$ term is valid at the first order. In a configuration such as the upstream Agulhas Current, the estimation of the $\overline{H R S}$ term using only the cross-front subterms is a workable approximation.

Submesoscale eddy generation by barotropic instability is a recurrent process when the Agulhas Current is not in a meandering state. The 1-year long numerical simulation provides the ability to check the impact of time-averaging on the cross-front $\overline{H R S}$ pattern. The 1-month average is defined in order to fit the SAGE's conditions: nonmeandering state, the mean front position along the $1000 \mathrm{~m}$ isobath, and submesoscale eddies developing. The 5-month average is instead defined as the consecutive months for which the current is not in a meandering state. Both time averages show similar patterns with slightly larger values for the 5-month average.

Submesoscale eddy generation by barotropic instability is a recurrent process along the Agulhas inshore front between $28^{\circ} \mathrm{E}$ and $26^{\circ} \mathrm{E}$. The $\overline{H R S}$ term averaged over a 5-month period (Figure 12) is very similar to the $\overline{H R S}$ term averaged over the 1-week period corresponding to the submesoscale eddy generation event of Figure 10. For both time periods, $\overline{H R S}$ has a very similar pattern with positive values $\left(O(2) \times 10^{-3} \mathrm{~m}^{3} \mathrm{~s}^{-3}\right)$ all along the front. Other energy conversion terms $\overline{V R S}$ and $\overline{V B F}$ have lower magnitudes (about $O(0.25) \times 10^{-3}$ $\mathrm{m}^{3} \mathrm{~s}^{-3}$ ) and have respectively negative and an alternation of negative and positive patterns along the front (not shown here). In addition to being a recurrent process, the generation of submesoscale eddies is the major process driving the local dynamics and variability of the Agulhas inshore front in a nonmeandering state in the vicinity of Port Elizabeth $\left(26^{\circ} \mathrm{E}\right)$. In the model, Natal Pulses inhibited the development of submesoscale eddies, but these mesoscale meanders can still host submesoscale activity following the mechanisms described by Gula et al. (2016a) in the context of Gulf Stream frontal eddies.

In this section, we confirm barotropic instability as the submesoscale eddy generation mechanism (Figures 9 and 10). The submesoscale eddies are recurrent features which locally drive the Agulhas frontal dynamics and variability (Figure 12). Comparisons between the SAGE data and the model show that they captured the same frontal dynamics despite their respective limitations (Figure 11). 


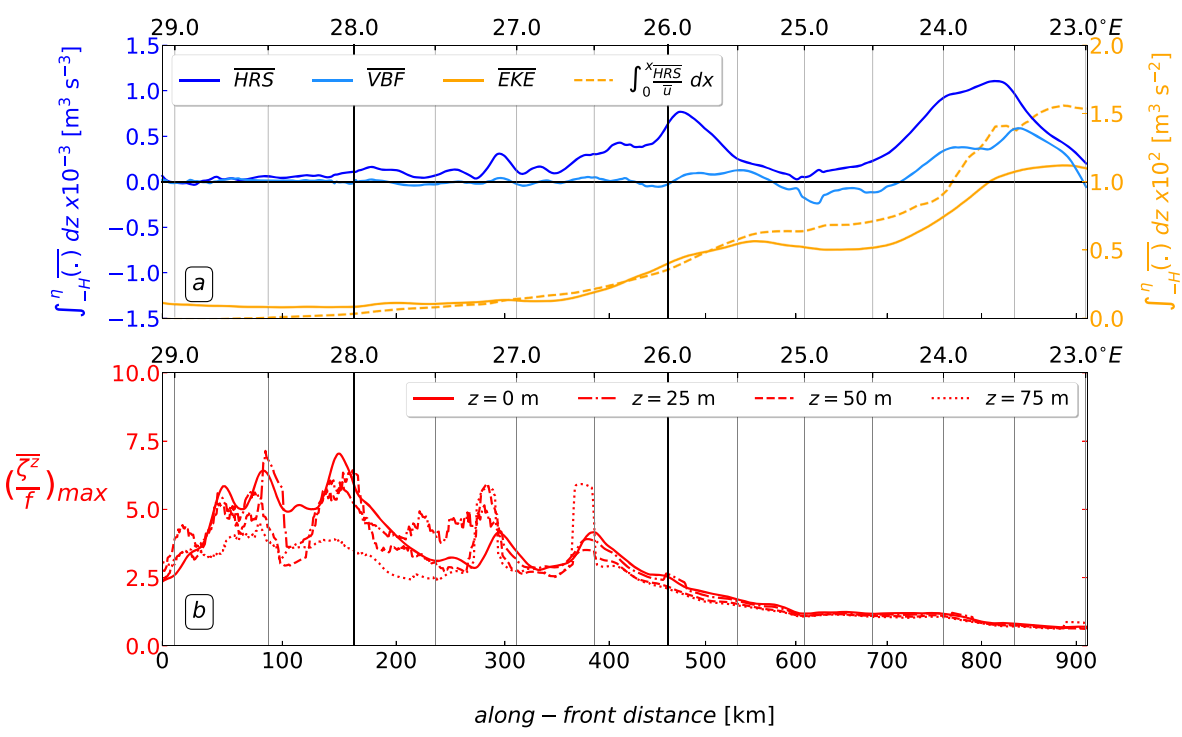

Figure 13. (a) Shows along-front distributions of the vertically integrated time mean energy conversion rate, $\overline{H R S}\left[\mathrm{~m}^{3}\right.$ $\left.\mathrm{s}^{-3}\right]$, time mean $E K E, \overline{E K E}\left[\mathrm{~m}^{3} \mathrm{~s}^{-2}\right]$ and $\int_{0}^{x} \frac{\overline{H R S}}{\bar{u}} \quad d x\left[\mathrm{~m}^{3} \mathrm{~s}^{-2}\right]$. The along-front distributions result from a cross-front average over the width (of about $100 \mathrm{~km}$ ) of the black dashed box centered on the inshore front shown in Figure 12. (b) Shows cross-front maximum of mean relative vorticity, $\frac{\bar{\zeta}}{f}$, along the Agulhas front at the surface, 25, 50 and $75 \mathrm{~m}$ depth. In both panels, the reading direction for the along-front distributions corresponds to the westward direction of the current. The significant increase of $\overline{H R S}$ and $\overline{E K E}$ between $28^{\circ} \mathrm{E}$ and $26^{\circ} \mathrm{E}$ reflects the submesoscale eddies generation. Upstream of this front portion, the relative vorticity is large indicating an unstable front.

\section{Spatial and Temporal Variability of the Instability}

In this section we aim to better characterize the barotropic instability spatial and time variability.

\subsection{Spatial Variability of the Instability}

\subsubsection{Where Does Barotropic Instability Occur Along the Agulhas Front ?}

To investigate where the barotropic instability is triggered along the front, we determine the contribution of $\overline{H R S}$ to the spatial evolution of $\overline{E K E}$. If $\overline{H R S}$ is the main source of $\overline{E K E}$ (Figures 10 and 12) 1011, the $\overline{E K E}$ equation (Equation 2) reduces at first order to:

$$
\overline{\frac{D E K E}{D t}} \equiv \bar{u} \partial_{x} \overline{E K E} \equiv \overline{H R S}
$$

with $u$ the along-front velocity component, $x$ the corresponding direction and the overbar denoting a 5-month time average when no Natal Pulse is present. The contribution of $\overline{H R S}$ to the along-front evolution of $\overline{E K E}$ is estimated from $\int_{0}^{x} \frac{\overline{H R S}}{\bar{u}} d x$ and compared to the full $\overline{E K E}$ (Figure 13a).

Even though the mean energy conversion rate $\overline{H R S}$ is positive over the Agulhas inshore front from $28^{\circ} \mathrm{E}$ to $23^{\circ} \mathrm{E}$ (Figures 10 and 12), the submesoscale eddies develop preferentially over a specific portion: between $28^{\circ} \mathrm{E}$ and $26^{\circ} \mathrm{E}$ (Figure 7). This specific portion is also visible from the along-front distributions of $\overline{E K E}$ and of the energy conversion rates $\overline{H R S}$ and $\overline{V B F}$ (Figure 13a).

Between $29^{\circ} \mathrm{E}$ and $25.5^{\circ} \mathrm{E}, \overline{H R S}$ is the dominant term responsible for the $\overline{E K E}$ generation and drives its along-front variations. The approximation $\overline{E K E} \approx \int_{0}^{x} \frac{\overline{H R S}}{\bar{u}} \quad d x$ is valid here. $\overline{H R S}$ has positive values almost monotonously increasing and the $\overline{E K E}$ is strongly increasing. Both patterns reflect the recurrent formation of vortex streets between $28^{\circ} \mathrm{E}$ and $26^{\circ} \mathrm{E}$ (Figure 7) and their downstream advection.

Westward of Port Elizabeth $\left(26^{\circ} \mathrm{E}\right)$, a combination of barotropic and baroclinic processes is responsible for the $\overline{E K E}$ generation, but they combine with other processes acting as sinks of $\overline{E K E}$. At first order, the evolution of $\overline{E K E}$ still follows the pattern of $\overline{H R S} \cdot \overline{H R S}$ decreases to zero from $26^{\circ} \mathrm{E}$ to $25^{\circ} \mathrm{E}$ and, as a result, $\overline{E K E}$ is flatter between $25.5^{\circ} \mathrm{E}$ and $24.5^{\circ} \mathrm{E}$. Downstream of $24.5^{\circ} \mathrm{E}, \overline{H R S}$ increases strongly again and $\overline{V B F}$ becomes positive and increases as well. Both terms reach a maximum at $23.5^{\circ} \mathrm{E}$. Accordingly, $\overline{E K E}$ increases strongly between $24.5^{\circ} \mathrm{E}$ and $23.5^{\circ} \mathrm{E}$. However, $\overline{E K E}$ does not increase as strongly as suggested by the $\overline{H R S}$ 
and $\overline{V B F}$ terms $\left(\overline{E K E}<\int_{0}^{x} \frac{\overline{H R S}+\overline{V B F}}{\bar{u}} d x\right)$ as other processes acting as $\overline{E K E}$ sinks are also at play. Sinks include effects of the bottom friction, wind stress, interior vertical mixing, horizontal diffusion, and of the nonlinear terms (Equation 2) advecting $\overline{E K E}$ out of the integration domain. Along this second front portion the flow still gains $\overline{E K E}$, but some is also strongly dissipated. This indicates a dynamical shift of the current towards a more turbulent nature.

This dynamical shift of the Agulhas current at $26^{\circ} \mathrm{E}$ is related to a topographical shift. The eastern margin of the Agulhas Bank, which begins westward of $26^{\circ} \mathrm{E}$, is associated with an abrupt topography change including a sudden widening of the shelf break and a curving of the topography (Figure 1). When reaching the eastern Agulhas Bank, the current is known to increasingly meander in the mesoscale range (Lutjeharms, 2006; Krug et al., 2014, 2009). This is reflected in the evolution of the vortex street. The modeled submesoscale eddies grow in size, potentially interact with each other and dissipate. Other perturbations, such as Shear-Edge eddies, are known to grow and occasionally leak downstream (Lutjeharms, 2006; Lutjeharms et al., 1989, 2003). To sum up, when flowing close to the topography (upstream of $26^{\circ} \mathrm{E}$ ) the barotropic instability leads to submesoscale eddy generation. When the Agulhas Current is in a nonmeandering state they are the only $\overline{E K E}$ source. When the topographic constraint relaxes on the current (downstream of $26^{\circ} \mathrm{E}$ ), the current develops meanders in the mesoscale range. The $\overline{E K E}$ sources are shared between barotropic and baroclinic processes and sinks also come into play.

The triggering of the barotropic instability between $28^{\circ} \mathrm{E}$ and $26^{\circ} \mathrm{E}$ is associated with intense relative vorticity upstream. Barotropic instability extracts energy from the mean horizontal shear. In the context of the upstream Agulhas Current, the main contribution to the horizontal shear is the lateral shear: $\partial_{y} \bar{u}$ (Figure 11). This term is also the main contributor to the current's relative vorticity. Cross-front maxima of relative vorticity, for different depths between the surface and $75 \mathrm{~m}$, are plotted along the Agulhas front on Figure 13b. The relative vorticity is more intense at the surface than at depth. It suggests that the front is more unstable at the very surface. This is coherent with $\overline{H R S}$ being more intense between the surface and $50 \mathrm{~m}$ depth (Figure 10 bottom panel) and the submesoscale eddies being surface-intensified (Figure 8 top panel).

The along-front relative vorticity provides an indication of where barotropic instability can be triggered and lead to submesoscale eddy generation. Upstream of the location of the barotropic instability triggering $\left(28^{\circ} \mathrm{E}\right)$, the relative vorticity is the largest, with values of about $O(6 f)$ at the surface, indicative of a potentially unstable front. Downstream of $28^{\circ} \mathrm{E}$, the relative vorticity decreases in two steps. Where the submesoscale eddies develop (between $28^{\circ} \mathrm{E}$ and $26^{\circ} \mathrm{E}$ ) the relative vorticity starts to decrease. Submesoscale eddies induce meanders in the front and in doing so they spread the mean frontal relative vorticity. The spread is the strongest at the surface where the submesoscale eddies are the most intense. Westward of $26^{\circ} \mathrm{E}$, the relative vorticity decreases, with values $O(<f)$, and becomes vertically homogeneous. This decrease is due to the formation of Agulhas frontal meanders of larger scales, downstream of Port Elizabeth $\left(26^{\circ} \mathrm{E}\right)$ where the topography constraint on the current is relaxed.

5.1.2. What Controls the Triggering of the Barotropic Instability Around $28^{\circ} \mathrm{E}$ ?

The barotropic instability originates from an unstable shear, itself set by the background strain. The mean normalized strain, $\bar{S} \times \frac{1}{f}$, is defined as

$$
\left(\begin{array}{l}
\partial_{x} \bar{u}-\partial_{y} \bar{v} \\
\partial_{x} \bar{v}+\partial_{y} \bar{u}
\end{array}\right)
$$

with $u$ and $v$ the velocity components in the $x$ and $y$ directions, $f$ the Coriolis parameter, and the overbar denoting a 5-month time average when no Natal Pulse is present. The background strain reflects the large scale straining and stretching acting on the flow. It can actively intensify the velocity and the horizontal gradients of tracers if its axis is aligned with the frontal axis. Both axes are aligned if their angle is inferior to $\frac{\pi}{4}$ (Hoskins, 1982), and in that case, the background strain is frontogenetic. Conversely, if the strain and the frontal axis are not aligned, the strain spreads the front and is frontolytic. The Agulhas inshore front is associated with an intense background strain at $25 \mathrm{~m}$ depth (Figure 14). Upstream of $28^{\circ} \mathrm{E}$, the strain is the strongest and is well aligned with the front, whereas downstream of $26^{\circ} \mathrm{E}$, it is weaker and the strain and frontal axis alignment is lost. The along-front distribution of the mean relative vorticity (Figure 13b) originates from the combined effect of the strain magnitude and of its alignment with the frontal axis. The 


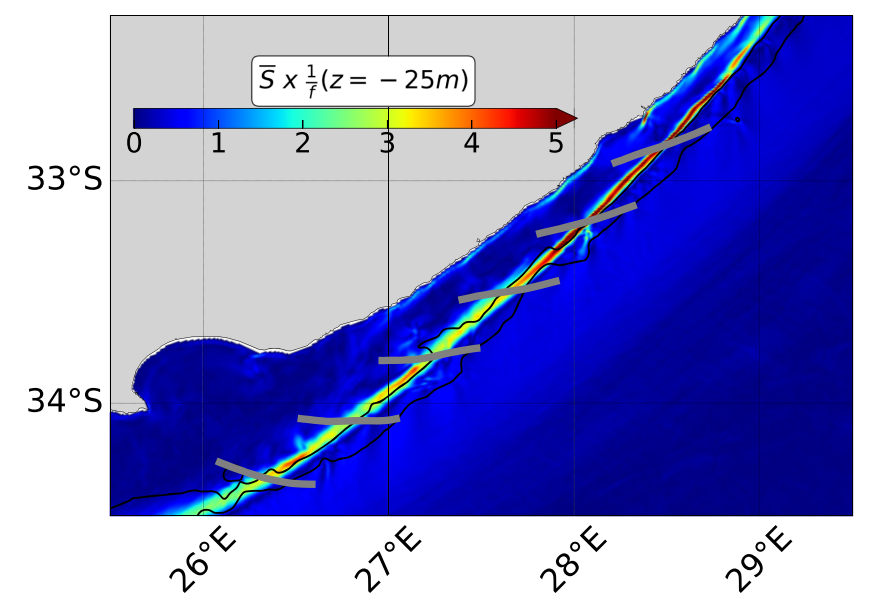

Figure 14. Map of the mean normalized background strain, $\bar{S} \times \frac{1}{f}$ at $25 \mathrm{~m}$. The overbar denotes a 5-month time average, when no Natal Pulse is present. Gray segments represent the strain axis and black contours the 200 and $1000 \mathrm{~m}$ isobaths. High relative vorticity, found upstream of the barotropic instability triggering $\left(28^{\circ} \mathrm{E}\right)$, corresponds to where the background strain is the most efficient at intensifying the front. The location of the barotropic instability triggering (between $28^{\circ} \mathrm{E}$ and $26^{\circ} \mathrm{E}$, Figure 7) corresponds to where the background strain relaxes its constraint on the front. portion where the front is the most sheared (upstream of $28^{\circ} \mathrm{E}$ ) is consistent with the background strain being the most efficient at sharpening and intensifying the Agulhas front.

The barotropic instability is triggered where the background strain weakens. Where the front destabilizes, downstream of $28^{\circ} \mathrm{E}$, the alignment of the strain with the front decreases, meaning the strain is less efficient at driving the front intensification. A frontogenetic background strain has the conjoint effect of intensifying the front and preventing mean horizontal shear-related instabilities to be triggered. In doing so it "stabilizes" a potentially unstable 2D relative vorticity band (Dritschel et al., 1991). Similarly, a frontogenetic tendency can prevent the triggering of shear instabilities on the upstream face of a Gulf Stream frontal eddy (Gula et al., 2016a), as well as the development of submesoscale cross-front disturbances on the upstream face of jet meandering troughs (McWilliams et al., 2019). By stretching and straining the front, the background strain exerts a stress in the along-front direction which inhibits any cross-front momentum and energy transfer. To sum up, the barotropic instability develops just downstream of a front portion where the relative vorticity is the most intense. This large relative vorticity originates from a frontogenetic background strain. The instability triggering is concomitant with the background strain weakening. When the front is released from the background strain constraint there is no external stress restraining the development of cross-frontal features and the front destabilizes.

A frontogenetic background strain usually results from the combination of the background mesoscale advection field and of the topographic constraint on the flow. Several studies connect front destabilizations with a weakening of the background strain (Gula et al., 2015b; Molemaker et al., 2015). In these studies, a straight and steep topography is identified to be at the origin of an intense background strain which is reinforced by the effect of the topographic drag on the current shear. An abrupt separation of the current from the topography leads to the strain weakening and thus to the triggering of barotropic instabilities just downstream the separation.

The Agulhas Current case differs slightly. The instability is triggered along a front portion where the current flows closely to the topography. In fact, the topographic constraint on the flow relaxes further downstream (at Port Elizabeth $26^{\circ} \mathrm{E}$ ). The triggering of the barotropic instability between $28^{\circ} \mathrm{E}$ and $26^{\circ} \mathrm{E}$ is thus not directly related to a topographic change. This mismatch between the front dynamics and the topographic constraint is also visible from the vertical structure of the strain (not shown here). Similarly to the relative vorticity (Figure 13b), the strain is the strongest at the surface even though along this portion of the front, the front is not in contact with the topography at depths shallower than 150-200 m (not shown here). Therefore, the background mesoscale advection field would be the main contributor to the intense background strain at the surface. The contribution of the topography in the front intensification and destabilization between $28^{\circ} \mathrm{E}$ and $26^{\circ} \mathrm{E}$ in the submesoscale range is less evident.

\subsection{Time Variability of the Instability}

While submesoscale eddy generation by barotropic instability is a recurrent process (Figures 11 and 12), which drives the Agulhas front variability in the submesoscale range in the vicinity of Port Elizabeth $\left(26^{\circ} \mathrm{E}\right)$, it is an intermittent process with a strong time variability.

The Agulhas front variability at relatively high frequencies is investigated with a five-month long time series of the energy conversion term HRS (Figure 15). The time series is built with the HRS term averaged over the vertical and over the frontal area between $28^{\circ} \mathrm{E}$ and $26^{\circ} \mathrm{E}$ from July to November when no Natal Pulse is present. Hence, this time series takes place during the austral winter, when Krug and Tournadre (2012) and Beal et al. (2015) observed the current to be less intense.

Submesoscale eddy generation shows a periodicity of about 10 days, on average, from the HRS time series. The HRS time series shows a constantly positive fluctuating signal (Figure 15). Not all the HRS peaks corre- 


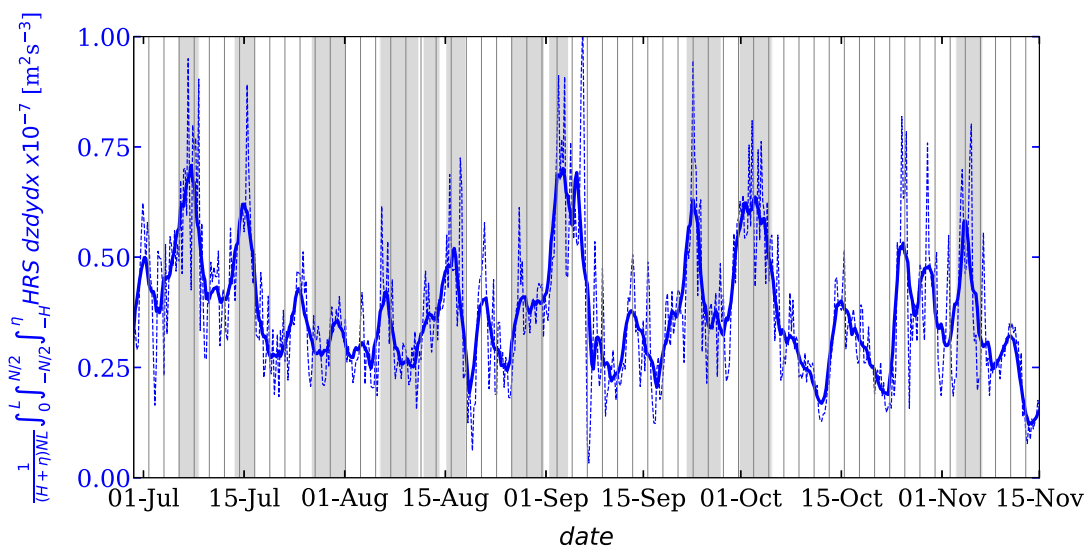

Figure 15. Time series of energy conversion rate $H R S$ over a 5 month-long period when no Natal Pulse is present. The time series results from a volume average: on the vertical, $H R S$ is averaged over the whole water column and on the horizontal over the $100 \mathrm{~km}$ wide black dashed box centered on the Agulhas front between $29^{\circ} \mathrm{E}$ and $26^{\circ} \mathrm{E}$ shown in Figure 12. The thin dashed and bold plain lines represent the raw and the smoothed (over a 10-point window) time series. The gray-shaded areas correspond to submesoscale eddies generation events identified from a movie a surface relative vorticity. Submesoscale eddies are generated when HRS is peaking, but the reverse is not true. This shows that a large $H R S$ is not a sufficient condition to trigger instabilities and that submesoscale eddies generation result from a combination of several factors.

spond to submesoscale eddy generation and ten events of actual submesoscale eddy generation are identified from a surface relative vorticity movie. They are marked in shades of gray on the HRS time series. These events are spaced by about 10 days on average. This periodicity also comes out from a 5-month long time series of the surface relative vorticity upstream of the instability triggering location $\left(28^{\circ} \mathrm{E}\right.$; not shown here). The triggering of the barotropic instability is then connected to the fluctuations, with a 10 days period, of the Agulhas inshore front structure.

The submesoscale eddy generation periodicity is relatively close to the Agulhas Current and to the Agulhas Undercurrent's ones. Bryden et al. (2005) and Beal (2009) respectively looked at the Agulhas Current and Agulhas Undercurrent time variability using 12 and a 17 month-long in situ records of current velocity. They show that both currents have the largest variability for the Natal Pulse period (60 days). At lower periods the Agulhas Current shows a large variability for periods between 8 and 27 days and the Agulhas Undercurrent for periods of 25 days and between 8 and 20 days. The variability of the submesoscale eddy generation is associated with large scale variations of the Agulhas Current and Undercurrent rather than with local variations of the Agulhas inshore front.

The few listed possible mechanisms involved in the time variability of the submesoscale eddy generation are a remote and a local forcings: the Coastal Trapped Waves or Rossby Topographic Waves and the wind stress respectively. Schumann and Brink (1990) showed, from in situ measurements of sea level along the Southern African coastline, that Coastal Trapped Waves affect the South African continental shelf dynamics and possibly interact with the Agulhas Current upstream of Port Elizabeth. Even though no study investigated the Rossby Topographic Waves along the South African continental slope, the location where the submesoscale eddies develop $\left(28^{\circ} \mathrm{E}-26^{\circ} \mathrm{E}\right)$ is favorable to their development in theory. Both waves can affect the Agulhas inshore front dynamics and then be involved or interact with the submesoscale eddies.

Rather than one process setting the conditions for the instability to be triggered, this can be the result of a combination of different processes all acting to destabilize the front. Local wind is another possible factor impacting the frontal dynamics. Thomas and Lee (2005) showed that down-front winds induce an Ekman transport of dense waters in the top of light waters resulting in the frontogenetic process enhancement. Schaeffer et al. (2017) observed the Eastern Australian Current mainly meandered and destabilized under up-front winds conditions leading to the formation of submesoscale frontal cyclonic eddies. However, in this study local wind forcing is not favorable before and during the submesoscale eddy generation. In this section we address the spatial and time variability of the barotropic instability triggering. The barotropic instability triggering in the submesoscale range and leading to the generation of frontal submesoscale eddies is the only 
source of variability for the Agulhas inshore front in the absence of Natal Pulses. The instability originates from an intense relative vorticity at the surface which itself is driven by the frontogenetic background strain. However, the relative contributions of the topography and of the background mesoscale advection field on driving the background strain are not completely disentangled and need further investigations. In the same way, further studies are needed to determine in which proportion remote and local mechanisms contribute to the 10 days variability of the instability triggering.

\section{Conclusion}

Our study addresses the Agulhas Current frontal dynamics in the submesoscale range. We investigated and characterized the generation mechanism of frontal submesoscale eddies along a nonmeandering Agulhas Current using a high-resolution $(\mathrm{dx} \sim 0.75 \mathrm{~km})$ numerical simulation. The simulation reproduces frontal cyclonic submesoscale eddies in the vicinity of Port Elizabeth $\left(26^{\circ} \mathrm{E}\right)$ with a diameter range of $10-20 \mathrm{~km}$ in agreement with satellite and in situ observations.

A one-dimensional linear stability analysis of the cross-front shear upstream of the instability triggering location $\left(28^{\circ} \mathrm{E}\right)$ shows that it is barotropically unstable and it develops perturbations with wavelengths of 10-13 km matching the scale of the submesoscale eddies. Barotropic instability is confirmed as the simulated submesoscale eddy generation mechanism by an energy transfer analysis. The barotropic instability is a horizontal shear instability which draws its energy from the mean kinetic energy field when the mean horizontal shear is unstable $(\overline{H R S}=M K E \rightarrow \overline{E K E})$. Comparison between the modeled barotropic instabilities and the ones observed during SAGE shows that the lateral shear is the main contribution to the energy conversion term $\overline{H R S}$.

Our energy transfer analysis over longer time periods (1 and 5-month long) indicates that the submesoscale frontal eddy generation by barotropic instability is a recurrent process which drives most of the variability around Port Elizabeth $\left(26^{\circ} \mathrm{E}\right)$.

This result gives new insights on the Agulhas Current along-stream dynamics. When considering a broader scale range, from the mesoscale to the submesoscale, the current dynamics are less dichotomous than previously described (Lutjeharms, 2006; Paldor \& Lutjeharms, 2009).

This study shows that in addition to the Natal Pulses (mesoscale solitary meanders), frontal submesoscale eddies also locally drive the "stable" branch variability around Port Elizabeth $\left(26^{\circ} \mathrm{E}\right)$. Rather than a dynamical shift, Port Elizabeth is the location of a smooth transition, between a "stable" and an "unstable" branch in the mesoscale range, via the triggering of instabilities in the submesoscale range. Hence, the so far known sources of variability for the upstream "stable" branch are the Natal Pulses and the frontal submesoscale eddies which both originate from barotropic instability (Beal et al., 2015; Biastoch \& Krauss, 1999; Tsugawa \& Hasumi, 2010).

The spatial variability of the barotropic instability triggering originates from the background strain. Between $28^{\circ} \mathrm{E}$ and $26^{\circ} \mathrm{E}$, barotropic instability is triggered and leads to the generation of frontal submesoscale eddies. Upstream of the instability triggering location $\left(28^{\circ} \mathrm{E}\right)$, the background strain is frontogenetic and the front is intensively sheared. The frontogenetic strain has the double effect of intensifying the shear and of preventing the development of cross-frontal perturbations. Downstream, the frontogenetic influence of the strain weakens allowing for barotropic instability to be triggered along the front.

The origin of the frontogenetic background strain slightly differs between the Agulhas Current and the Gulf Stream. The bottom friction does not play a leading role in generating the current's horizontal shear and the topographic constraint role is not as evident as in the Gulf Stream (Gula et al., 2015b).

The triggering of barotropic instability has a periodicity of about 10 days on average. Possible mechanisms driving this time variability are Coastal Trapped Waves or Rossby Topographic Waves and wind stress. Their relative contributions need further investigation.

The submesoscale frontal eddies could drive exchanges of material and momentum between shelf waters and the Agulhas Current, with potential consequences on coastal dynamics, marine life, and large scale circulation. Additional observations and modeling studies are required to address these aspects. 


\section{Acknowledgments}

This work was granted access to the HPC resources of IDRIS under the allocation A0040107630 made by GENCI at Paris, France, and of the HPC facilities DATARMOR of "Pôle de Calcul Intensif pour la Mer" at Ifremer, Brest, France. Model outputs are available upon request. This work was supported by the RADII funding as well as Ifremer and the Brittany region for $\mathrm{PhD}$ funding. Odyssea Regional South Africa products are available at http://www.ifremer.fr/opendap/ cerdap1/ghrsst/14/saf/odyssea-nrt/ data and MODIS SST data are available (Level 4) at http://cersat.ifremer.fr/ data/products/catalogue. The drifter datasets ANDRO and NOAA are respectively available at https://www. umr-lops.fr/Donnees/ANDRO and https://www.aoml.noaa.gov/phod/ gdp/interpolated/data/subset.php. The AVISO data are available at ftp.aviso. altimetry.fr. Gridded cross-sectional velocities are based on in situ current meter measurements from the Agulhas Current Time-series (ACT) experiment (Beal et al., 2015) and can be downloaded at https://beal-agulhas rsmas.miami.edu/data-and-products/ index.html. We, as well, want to especially thank Heather Reagan for her precious help in correcting the manuscript language.

\section{References}

Beal, L. (2009). A time series of Agulhas Undercurrent transport. Journal of Physical Oceanography, 39(10), $2436-2450$.

Beal, L., De Ruijter, W., Biastoch, A., \& Rainer, Z. (2011). On the role of the Agulhas system in ocean circulation and climate. Nature, 472 , 429-436.

Beal, L., Elipot, S., Houk, A., \& Leber, G. (2015). Capturing the transport variability of a western boundary jet: Results from the Agulhas Current Time-Series Experiment (ACT). Journal of Physical Oceanography, 45(5), 1302-1324.

Beckmann, A., \& Haidvogel, D. (1993). Numerical simulation of flow around a tall isolated seamount. Part I: Problem formulation and model accuracy. Journal of Physical Oceanography, 23(8), 1736-1753.

Biastoch, A., \& Krauss, W. (1999). The role of mesoscale eddies in the source regions of the Agulhas Current. Journal of Physical Oceanography, 29(9), 2303-2317.

Bryden, H., Beal, L., \& Duncan, L. (2005). Structure and transport of the Agulhas Current and its temporal variability. Journal of Oceanography, 61(3), 479-492.

Chelton, D., Deszoeke, R., Schlax, M., Naggar, K. E., \& Siwertz, N. (1998). Geographical variability of the first baroclinic Rossby radius of deformation. Journal of Physical Oceanography, 28(3), 433-460.

Chelton, D., Schlax, M., \& Samelson, R. (2011). Global observations of nonlinear mesoscale eddies. Progress in oceanography, 91(2), 167-216.

Debreu, L., Marchesiello, P., Penven, P., \& Chambon, G. (2012). Two-way nesting in split-explicit ocean models: Algorithms, implementation and validation. Ocean Modelling, 49-50, 1-21.

Dee, D. P., Uppala, S. M., Simmons, A. J., Berrisford, P., Poli, P., Kobayashi, S., et al. (2011). The ERA-Interim reanalysis: Configuration and performance of the data assimilation system. Quarterly Journal of the Royal Meteorological Society, 137(656), 553-597.

Dritschel, D., Haynes, P., Juckes, M., \& Shepherd, T. (1991). The stability of a two-dimensional vorticity filament under uniform strain. Journal of Fluid Mechanics, 230, 647-665.

Durski, S., Glenn, S., \& Haidvogel, D. (2004). Vertical mixing schemes in the coastal ocean: Comparison of the level 2.5 Mellor-Yamada scheme with an enhanced version of the K profile parameterization. Journal of Geophysical Research, 109, C01015. https://doi.org/10. 1029/2002JC001702

Elipot, S., \& Beal, L. M. (2015). Characteristics, energetics, and origins of Agulhas Current meanders and their limited influence on ring shedding. Journal of Physical Oceanography, 45(9), 2294-2314. https://doi.org/10.1175/JPO-D-14-0254.1

Fairall, C., Bradley, E., Rogers, D., Edson, J., \& Young, G. (1996). Bulk parameterization of air-sea fluxes for tropical ocean-global atmosphere coupled-ocean atmosphere response experiment. Journal of Geophysical Research, 101(C2), 3747-3764.

Ferrari, R., \& Wunsch, C. (2010). The distribution of eddy kinetic and potential energies in the global ocean. Tellus A: Dynamic Meteorology and Oceanography, 62(2), 92-108.

Ferry, N., Parent, L., Garric, G., Bricaud, C., Testut, C. E., Le Galloudec, O., et al. (2012). GLORYS2V1 global ocean reanalysis of the altimetric era (1992-2009) at meso scale. Mercator Ocean Quaterly Newsletter, 44, 29-39.

Gula, J., Molemaker, M., \& McWilliams, J. (2014). Submesoscale cold filaments in the Gulf Stream. Journal of Physical Oceanography, 44(10), 2617-2643.

Gula, J., Molemaker, M., \& McWilliams, J. (2015a). Gulf Stream dynamics along the Southeastern U.S. Seaboard. Journal of Physical Oceanography, 45(3), 690-715.

Gula, J., Molemaker, M., \& McWilliams, J. (2015b). Topographic vorticity generation, submesoscale instability and vortex street formation in the Gulf Stream. Geophysical Research Letters, 42, 4054-4062. https://doi.org/10.1002/2015GL063731

Gula, J., Molemaker, M., \& McWilliams, J. (2016a). Submesoscale dynamics of a Gulf Stream frontal eddy in the South Atlantic Bight. Journal of Physical Oceanography, 46(1), 305-325.

Gula, J., Molemaker, M., \& McWilliams, J. (2016b). Topographic generation of submesoscale centrifugal instability and energy dissipation. Nature communications, 7, 12811.

Haidvogel, D. B., \& Beckmann, A. (1999). Numerical ocean circulation modeling.

Halo, I., Backeberg, B., Penven, P., Ansorge, I., Reason, C., \& Ullgren, J. (2014). Eddy properties in the Mozambique Channel: A comparison between observations and two numerical ocean circulation models. Deep Sea Research Part II: Topical Studies in Oceanography, $100,38-53$.

Hoskins, B. J. (1982). The mathematical theory of frontogenesis. Annual Review of Fluid Mechanics, 14(1), 131-151.

Krug, M., Swart, S., \& Gula, J. (2017). Submesoscale cyclones in the Agulhas Current. Geophysical Research Letters, 44, 346-354. https:// doi.org/10.1002/2016GL071006

Krug, M., \& Tournadre, J. (2012). Satellite observations of an annual cycle in the Agulhas Current. Geophysical Research Letters, 39 , L15607. https://doi.org/10.1029/2012GL052335

Krug, M., Tournadre, J., \& Dufois, F. (2014). Interactions between the Agulhas Current and the eastern margin of the Agulhas Bank. Continental Shelf Research, 81, 67-79.

Large, W. G., McWilliams, J. C, \& Doney, S. C. (1994). Oceanic vertical mixing: A review and a model with a nonlocal boundary layer parameterization. Reviews of Geophysics, 32(4), 363-403.

Lemarié, F., Kurian, J., Shchepetkin, A., Molemaker, M., Colas, F., \& McWilliams, J. (2012). Are there inescapable issues prohibiting the use of terrain-following coordinates in climate models? Ocean Modelling, 42, 57-79.

Lutjeharms, J. (2006). The agulhas current (Vol. 5). Berlin: Springer.

Lutjeharms, J., Catzel, R., \& Valentine, H. (1989). Eddies and other boundary phenomena of the Agulhas Current. Continental Shelf Research, 9(7), 597-616.

Lutjeharms, J., \& Gordon, A. (1987). Shedding of an Agulhas ring observed at sea. Nature, 325(6100), 138

Lutjeharms, J., Penven, P., \& Roy, C. (2003). Modelling the shear edge eddies of the southern Agulhas Current. Continental Shelf Research, 23(11-13), 1099-1115.

Mahadevan, A. (2014). Ocean science: Eddy effects on biogeochemistry. Nature, 506(7487), 168.

Marchesiello, P., Debreu, L., \& Couvelard, X. (2009). Spurious diapycnal mixing in terrain-following coordinate models: The problem and a solution. Ocean Modelling, 26(3-4), 156-169.

McWilliams, J. C., Gula, J., \& Molemaker, M. J. (2019). The Gulf Stream North Wall: Ageostrophic circulation and frontogenesis. Journal of Physical Oceanography, 49(4), 893-916.

Ménesguen, C., McWilliams, J., \& Molemaker, M. (2012). Ageostrophic instability in a rotating stratified interior jet. Journal of Fluid Mechanics, 711, 599-619. 
Molemaker, M., McWilliams, J., \& Dewar, W. (2015). Submesoscale instability and generation of mesoscale anticyclones near a separation of the California Undercurrent. Journal of Physical Oceanography, 45(3), 613-629.

Paldor, N., \& Lutjeharms, J. (2009). Why is the stability of the Agulhas Current geographically bi-modal? Geophysical Research Letters, 36, L14604. https://doi.org/10.1029/2009GL038445

Peeters, F., Acheson, R., Brummer, G. J., De Ruijter, W., Schneider, R., Ganssen, G., et al. (2004). Vigorous exchange between the Indian and Atlantic oceans at the end of the past five glacial periods. Nature, 430(7000), 661.

Penven, P., Lutjeharms, J., Marchesiello, P., Roy, C., \& Weeks, S. (2001). Generation of cyclonic eddies by the Agulhas Current in the Lee of the Agulhas Bank. Geophysical Research Letters, 28(6), 1055-1058. https://doi.org/10.1029/2000GL011760

Renault, L., McWilliams, J., \& Penven, P. (2017). Modulation of the Agulhas Current retroflection and leakage by oceanic current interaction with the atmosphere in coupled simulations. Journal of Physical Oceanography, 47(8), 2077-2100.

Ridgway, K. R., Dunn, J. R., \& Wilkin, J. L. (2002). Ocean interpolation by four-dimensional weighted least squares-Application to the waters around Australasia. Journal of Atmospheric and Oceanic Technology, 19(9), 1357-1375.

Rouault, M., \& Penven, P. (2011). New perspectives on Natal Pulses from satellite observations. Journal of Geophysical Research, 116, C07013. https://doi.org/10.1029/2010JC006866

Schaeffer, A., Gramoulle, A., Roughan, M., \& Mantovanelli, A. (2017). Characterizing frontal eddies along the East Australian Current from HF radar observations. Journal of Geophysical Research: Oceans, 122, 3964-3980. https://doi.org/10.1002/2016JC012171

Schouten, M., De Ruijter, W., \& Van Leeuwen, P. (2002). Upstream control of Agulhas Ring shedding. Journal of Geophysical Research, 107(C8), 3109. https://doi.org/10.1029/2001JC000804

Schumann, E., \& Brink, K. (1990). Coastal-trapped waves off the coast of South Africa: Generation, propagation and current structures. Journal of Physical Oceanography, 20(8), 1206-1218.

Schumann, E., \& Van Heerden, I. (1988). Observations of Agulhas Current frontal features south of Africa, October 1983. Deep Sea Research Part A. Oceanographic Research Papers, 35(8), 1355-1362.

Shchepetkin, A., \& McWilliams, J. (2005). The Regional Oceanic Modeling System (ROMS): A split-explicit, free-surface, topography-following- coordinate ocean model. Ocean Modelling, 9, 347-404.

Soufflet, Y., Marchesiello, P., Lemarié, F., Jouanno, J., Capet, X., Debreu, L., \& Benshila, R. (2016). On effective resolution in ocean models. Ocean Modelling, 98, 36-50.

Thomas, L., \& Lee, C. (2005). Intensification of ocean fronts by down-front winds. Journal of Physical Oceanography, 35(6), 1086-1102.

Tsugawa, M., \& Hasumi, H. (2010). Generation and growth mechanism of the Natal Pulse. Journal of Physical Oceanography, 40(7), 1597-1612.

Weatherall, P., Marks, K. M., Jakobsson, M., Schmitt, T., Tani, S., Arndt, J. E., et al. (2015). A new digital bathymetric model of the world's oceans. Earth and Space Science, 2(8), 331-345.

Weijer, W., De Ruijter, W., Sterl, A., \& Drijfhout, S. (2002). Response of the Atlantic Overturning Circulation to South Atlantic sources of buoyancy. Global and Planetary Change, 34(3-4), 293-311. 